\title{
Intranasal siRNA administration reveals IGF2 deficiency contributes to impaired cognition in Fragile $X$ syndrome mice
}

\author{
Marta Pardo, ${ }^{1,2}$ Yuyan Cheng, ${ }^{1,2}$ Dmitry Velmeshev, ${ }^{1}$ Marco Magistri,, ${ }^{1}$ Hagit Eldar-Finkelman, ${ }^{3}$ \\ Ana Martinez, ${ }^{4}$ Mohammad A. Faghihi, ${ }^{1}$ Richard S. Jope, ${ }^{1,2}$ and Eleonore Beurel ${ }^{1,2}$ \\ 'Department of Psychiatry and Behavioral Sciences, ${ }^{2}$ Department of Biochemistry and Molecular Biology, Miller School of \\ Medicine, University of Miami, Miami, Florida, USA. ${ }^{3}$ Department of Human Molecular Genetics and Biochemistry, Sackler \\ School of Medicine, Tel Aviv University, Tel Aviv, Israel. ${ }^{4}$ Centro de Investigaciones Biologicas-CSIC, Madrid, Spain.
}

\begin{abstract}
Molecular mechanisms underlying learning and memory remain imprecisely understood, and restorative interventions are lacking. We report that intranasal administration of siRNAs can be used to identify targets important in cognitive processes and to improve genetically impaired learning and memory. In mice modeling the intellectual deficiency of Fragile $X$ syndrome,

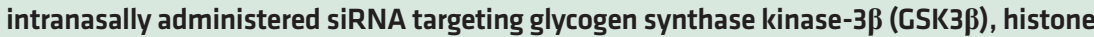
deacetylase-1 (HDAC1), HDAC2, or HDAC3 diminished cognitive impairments. In WT mice, intranasally administered brain-derived neurotrophic factor (BDNF) siRNA or HDAC4 siRNA impaired learning and memory, which was partially due to reduced insulin-like growth factor-2 (ICF2) levels because the BDNF siRNA- or HDAC4 siRNA-induced cognitive impairments were ameliorated by intranasal IGF2 administration. In Fmr1--- mice, hippocampal IGF2 was deficient, and learning and memory impairments were ameliorated by IGF2 intranasal administration. Therefore intranasal siRNA administration is an effective means to identify mechanisms regulating cognition and to modulate therapeutic targets.
\end{abstract}

Authorship note: RSJ and EB contributed equally to this work.

Conflict of interest: The authors have declared that no conflict of interest exists.

Submitted: November 28, 2016

Accepted: February 8, 2017

Published: March 23, 2017

Reference information: JCI Insight. 2017;2(6):e91782. https:// doi.org/10.1172/jci.insight.91782

\section{Introduction}

Many potentially effective drugs have been developed for treating various aspects of neurological and psychiatric diseases, such as for improving impairments in learning and memory, but translation faces the difficult obstacle of attaining sufficient delivery to the CNS. Inhibitors of glycogen synthase kinase-3 (GSK3) and inhibitors of histone deacetylases (HDACs) are 2 classes of such drugs with potential to ameliorate cognitive impairments.

GSK 3 refers to 2 isoforms, GSK $3 \alpha$ and $\operatorname{GSK} 3 \beta(1,2)$. GSK 3 is primarily regulated by phosphorylation of an N-terminal serine in each of its 2 isoforms, serine- 21 in GSK3 $\alpha$ and serine- 9 in GSK3 $\beta$. The effects of abnormally active GSK3 has been studied in GSK3 knockin mice in which the inhibitory serines are mutated to alanines, leaving GSK3 constitutively active at a physiologically relevant level because GSK3 is not overexpressed (3). GSK3 is a central hub in several intracellular signaling pathways (1). Two of these include inhibition by GSK3 of long-term potentiation and promotion of long-term depression, 2 processes that are critical for learning and memory $(4,5)$. The therapeutic potential of GSK3 inhibitors for ameliorating impairments in learning and memory has been demonstrated in a several rodent models (reviewed in ref. 6), such as Fragile X syndrome (7) and Alzheimer's disease (8). However, therapeutic applications in patients have been hampered by the difficulty in obtaining CNS levels of GSK3 inhibitors sufficient to be therapeutic without side effects due to inhibition of GSK3 in peripheral organs (8).

There is also increasing evidence that HDACs modify learning and memory, initially demonstrated by the beneficial effects of general HDAC inhibitors on learning and memory in rodent models (9-14). Examining which subtypes of HDACs modulate learning, mouse forebrain overexpression of HDAC2, but not HDAC1, was found to impair memory, whereas memory was facilitated by HDAC2 deficiency or administration of HDAC inhibitors (15). Deleterious effects of HDAC2 on memory were further supported by subsequent studies (16-18). Similarly to HDAC2 modulation, deletion of HDAC3 in area CA1 of the dorsal hippocampus or administration of an HDAC inhibitor with a preferential effect on 
HDAC3 enhanced memory, demonstrating detrimental effects of HDAC3 (19). In contrast, HDAC4 promotes synaptic plasticity and memory formation $(20,21)$. These findings suggest that specific inhibitors of subtypes of HDACs may be useful to modulate learning and memory, but in spite of a concerted effort, there remains limited progress in identifying CNS-penetrant, HDAC subtype-specific inhibitors capable of ameliorating learning deficits $(22,23)$.

One method to achieve specific inhibition of targets that regulate learning is the use of siRNA. However, this potential treatment for impaired learning also faces the major hurdle of attaining effective delivery to the CNS, which could provide a specific means to knock down the expression of targets involved in CNS diseases $(24,25)$. Several reports showed that nanoparticle-linked siRNA can be delivered into the brain by intranasal administration to knock down target proteins $(26,27)$. We embarked on an investigation using this approach to target GSK3 $\beta$ as a potential therapeutic intervention for the severe intellectual disability of Fragile X syndrome (28). For this, we used 2 mouse models of Fragile X syndrome that display impaired cognition, $F m r 1^{-1-}$ mice and GSK3 knockin mice, because GSK3 is abnormally active in $\mathrm{Fmrl}^{\mathrm{I}^{-1}}$ mouse hippocampus and other regions, and peripheral administration of GSK3 inhibitors ameliorate several impairments in learning and memory in $F m r 1^{-/-}$mice (29-31). As a control for nanoparticle-coupled GSK3 $\beta$ siRNA, we intranasally administered noncoupled GSK3 $\beta$ siRNA. We were surprised to find that GSK3 $\beta$ in mouse hippocampus was significantly reduced by this approach. Here, we report that impairments in learning and memory in $\mathrm{Fmrl}^{-/-}$mice can be ameliorated by intranasal administration of either a GSK3 inhibitor or GSK3 $\beta$ siRNA. Furthermore, we report that intranasal application of siRNA can be used to identify molecules required for learning in mice, as well as to treat learning and memory impairments. Using intranasal siRNA administration, we identified subtypes of HDACs that repair or impair learning and memory, and we identified insulin-like growth factor-2 (IGF2) as a key regulator of learning and memory in $\mathrm{Fmrl}^{-/-}$mice.

\section{Results}

Intranasal administration of a GSK3 $\beta$ inhibitor or siRNA ameliorates learning and memory impairments in Fmr1 ${ }^{-1}$ mice. We first tested if intranasal administration of a GSK3 inhibitor, L803-mts (32), could improve 4 measures of learning and memory that are impaired in $\mathrm{Fmrl}^{-/-}$mice to test if this route of administration can impact regions relevant for cognition $(30,31)$. Impairments in $\mathrm{Fmrl}^{-/-}$mice were corrected by intranasal administration of L803-mts in novel object recognition (Figure 1A), temporal ordering (Figure 1B), and coordinate (Figure 1C) and categorical (Figure 1D) spatial processing. L803-mts administration did not significantly alter the behavior of WT mice in these processes (Figure 1, A-D). Thus, intranasal administration of a GSK3 inhibitor is sufficient to improve 4 measures of learning and memory in $\mathrm{Fmrl}^{\mathrm{I}^{--}}$mice.

We then tested if intranasal administration of GSK3 $\beta$ siRNA could match the therapeutic effects of L803-mts. Cognitive deficits in $\mathrm{Fmrl}^{-1-}$ mice were rescued by intranasal administration of either of 2 different GSK3 $\beta$ siRNA sequences, but not by scrambled siRNA, in novel object recognition (Figure 1E), temporal ordering (Figure $1 \mathrm{~F}$ ), and coordinate (Figure $1 \mathrm{G}$ ) and categorical (Figure $1 \mathrm{H}$ ) spatial processing. Intranasal GSK3 $\beta$ siRNA administration did not alter the performance of WT mice in any of these measures (Figure 1, E-H). Intranasal administration of scrambled siRNA coupled to fluorescein revealed fluorescein accumulation in the hippocampus but not in the cerebellum (Figure 1I). Intranasal administration of GSK3 $\beta$ siRNA caused a significant reduction of GSK3 $\beta$ levels in the hippocampus (Supplemental Figure 1, A-S; supplemental material available online with this article; https://doi.org/10.1172/jci. insight.91782DS1). Although we focused on the hippocampus in this study, we also tested if another region was affected by intranasal administration of GSK3 $\beta$ siRNA and found reduced GSK3 $\beta$ in the perirhinal cortex (Supplemental Figure 1, T-Z). Further studies are required to test if other brain regions involved in learning and memory are also impacted by intranasal siRNA administration. Altogether, these results reveal that intranasal siRNA administration is as efficacious as pharmacological inhibition of GSK3 to functionally modulate learning and memory in genetically impaired mice.

Intranasal administration of BDNF siRNA impairs learning and memory in WT mice. To further validate the methodology of using intranasal siRNA administration to modulate learning and memory, we tested if targeting a molecule known to be required for learning and memory is capable of inducing cognitive impairments in WT mice. For this, we used brain-derived neurotrophic factor (BDNF) siRNA, since BDNF is required for learning and memory $(33,34)$. Intranasal administration of BDNF siRNA, but not scrambled siRNA, to WT mice dose-dependently impaired novel object recognition (Figure 2, A and B), 
A

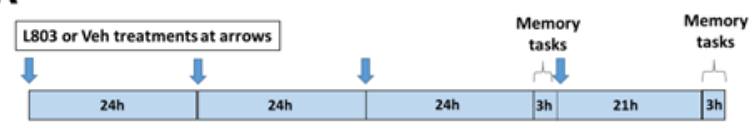

Novel object recognition

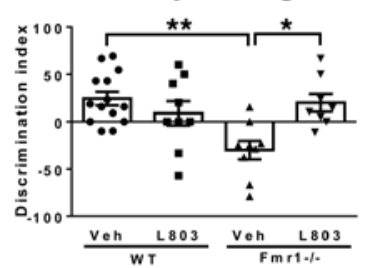

B Temporal order recognition
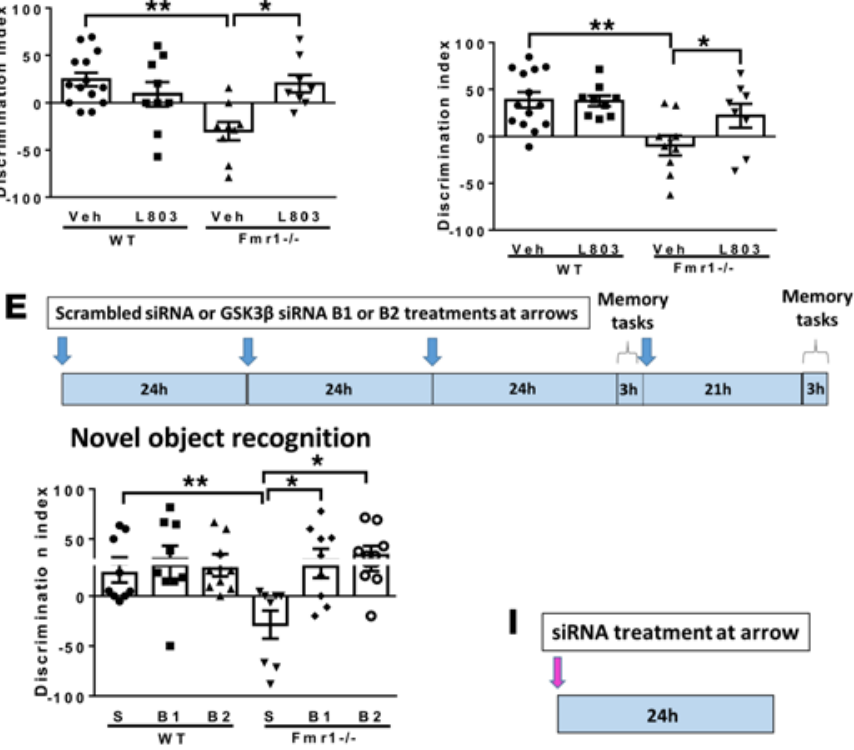

H Coordinate spatial processing

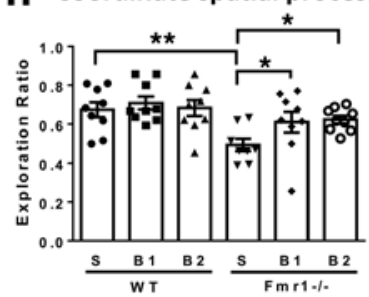

I
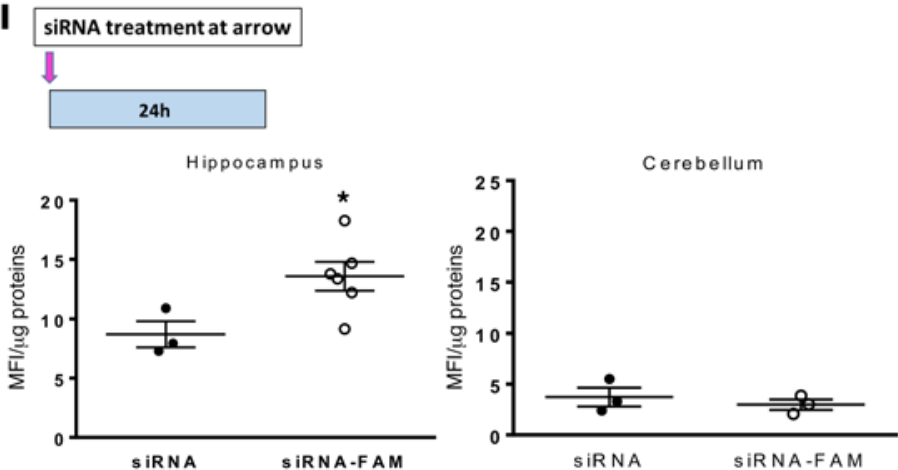

D Categorical spatial processing
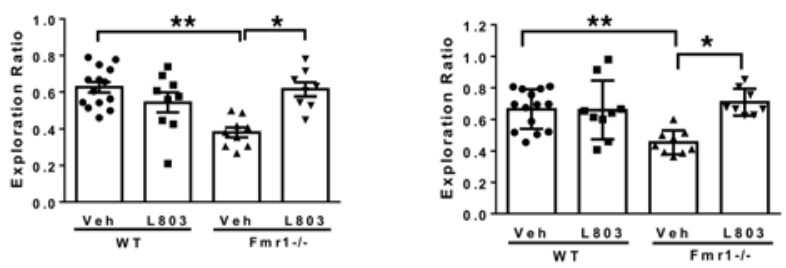

F Temporal order recognition
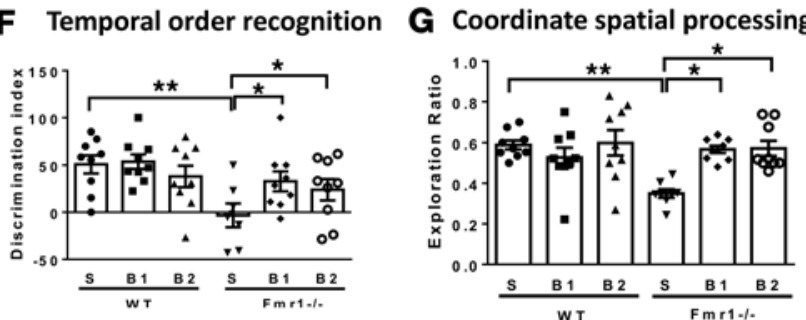

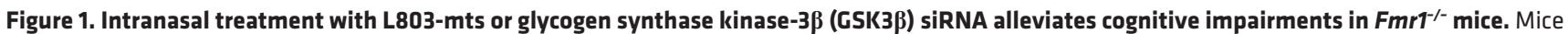
were treated intranasally with the GSK3 inhibitor L803-mts or with GSK3 $\beta$ siRNA followed by 4 measures of learning and memory (novel object recognition, temporal ordering, and coordinate and categorical spatial processing) to test if cognition was improved in $\mathrm{Fmr1}^{-/-}$mice, which model Fragile $X$ syndrome and are known to display cognitive impairments, or if cognition was affected in WT mice. WT and Fmr1 ${ }^{-/-}$mice received (A-D) intranasal vehicle (Veh) or L803-mts (L803; $60 \mu \mathrm{g} /$ mouse), or (E-H) intranasal scrambled siRNA (S) or 2 different sequences of GSK3 $\beta$ siRNA (B1, sequence 1, or B2, sequence $2 ; 10 \mu \mathrm{g} / \mathrm{mouse} / \mathrm{day}$ ) daily for 3 consecutive days prior to testing and daily throughout the behavioral testing. Discrimination index is shown for (A) novel object recognition $\left(n=8-14 ; \mathrm{F}_{(3,39)}=6.49, P<0.01\right)\left({ }^{* *} P<0.01\right.$ compared with vehicle-treated WT mice; ${ }^{*} P<0.01$ compared with vehicle-treated Fmr1 ${ }^{-1-}$ mice) and (B) temporal ordering $\left(n=8-14 ; \mathrm{F}_{(3,39)}=5.87, P<0.01\right)\left({ }^{* *} P<0.01\right.$ compared with vehicle-treated WT mice; ${ }^{*} P<0.05$ compared with vehicle-treated $F m r 1^{-1-}$ mice). Exploration ratio is shown for (C) coordinate spatial processing $\left(n=8-14 ; F_{(3.39)}=9.07, P<0.01\right)\left({ }^{* *} P<0.01\right.$ compared with vehicle-treated WT mice; ${ }^{*} P<0.01$ compared with vehicle-treated $F m r 1^{-1-}$ mice) and (D) categorical spatial processing $\left(n=8-14 ; \mathrm{F}_{(3,39)}=7.38, P<0.01\right)\left({ }^{* *} P\right.$ $<0.01$ compared with vehicle-treated WT mice; ${ }^{*} P<0.01$ compared with vehicle-treated $\mathrm{Fmr}^{-1-}$ mice). Discrimination index is shown for (E) novel object recognition $\left(n=7-9 ; F_{(5,52)}=4.34, P<0.01\right)\left({ }^{* *} P<0.01\right.$ compared with scrambled siRNA-treated WT mice; $P<0.01$ compared with scrambled siRNAtreated $\mathrm{Fmr1}^{-/-}$mice) and $(\mathbf{F})$ temporal ordering $\left(n=7-9 ; \mathrm{F}_{(5,51)}=3.51, P<0.01\right)\left({ }^{* *} P<0.01\right.$ compared with scrambled siRNA-treated WT mice; ${ }^{*} P<0.05$ compared with scrambled siRNA-treated $F m r 1^{-1-}$ mice). Exploration ratio is shown for (C) coordinate spatial processing $\left(n=7-9 ; F_{(5,52)}=5.42, P<0.01\right)(* * P$ $<0.01$ compared with scrambled siRNA-treated WT mice; ${ }^{*} P<0.01$ compared with scrambled siRNA-treated Fmr1 ${ }^{-1-}$ mice) and (H) categorical spatial processing $\left(n=7-9 ; F_{(5.52)}=3.89, P<0.01\right)\left({ }^{* *} P<0.01\right.$ compared with scrambled siRNA-treated WT mice; ${ }^{*} P<0.01$ compared with scrambled siRNA-treated $\mathrm{Fmr1}^{-1-}$ mice). One-way ANOVA (genotype $\times$ treatment) for each behavioral test followed by post hoc Dunnett's multiple comparison test. (I) Mice received intranasally scrambled siRNA coupled or not to fluorescein (FAM), and 24 hours after injection, mean fluorescence intensity (MFI) was measured in the hippocampus and the cerebellum. ${ }^{*} P<0.05$ (Student's $t$ test). Each symbol represents the value from an individual mouse. Values are means \pm SEM.

coordinate spatial processing (Figure 2C), and categorical spatial processing (Figure 2D). These results demonstrate that intranasal administration of siRNA for a molecule known to be critical for learning and memory can indeed induce cognitive impairments in WT mice.

Intranasal administration of HDAC siRNAs modulate learning and memory. Current knowledge of the roles of HDAC subtypes in regulating learning and memory is largely based on the effects of overexpression and deletion. Therefore, we tested if siRNAs targeting subtypes of HDACs could provide a means to reg- 
A

\section{Veh or BDNF siRNA treatment at arrow}

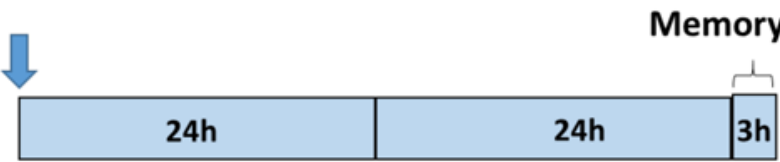

Novel object recognition

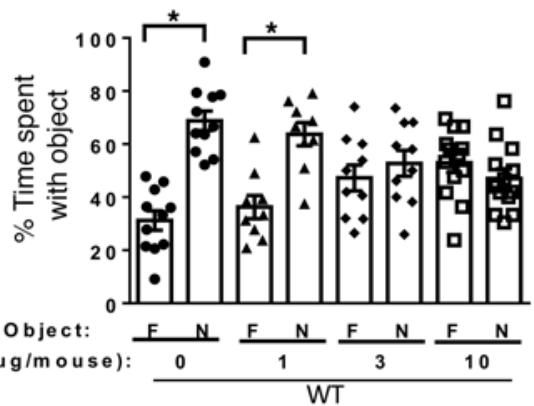

C Coordinate spatial processing

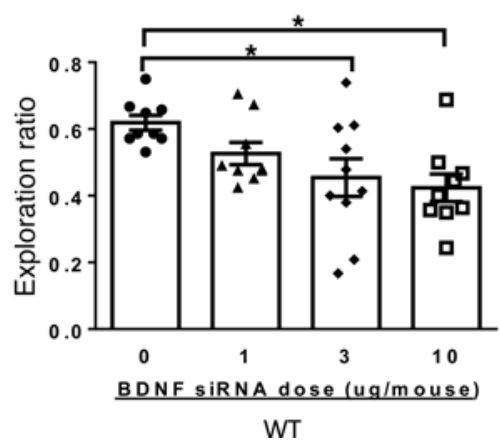

B Novel object recognition

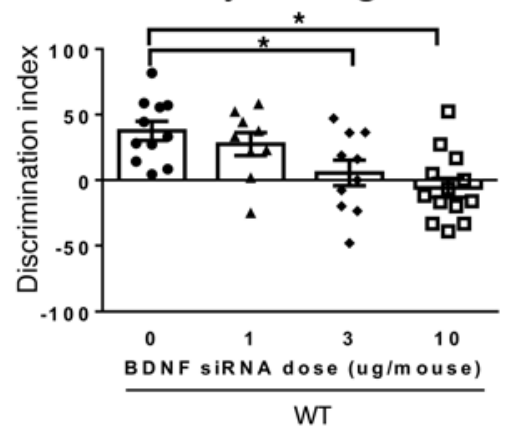

D Categorical spatial processing

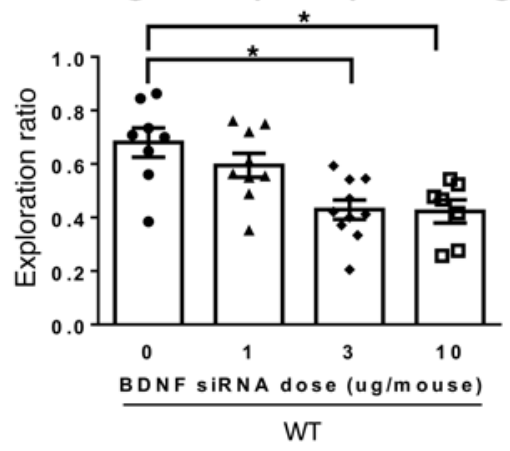

Figure 2. Intranasal treatment with brain-derived neurotrophic factor (BDNF) siRNA impairs learning and memory. To validate the methodology of using intranasal siRNA administration to modulate learning and memory, we tested if targeting BDNF, which is known to be required for learning and memory, is capable of inducing cognitive impairments in WT mice in novel object recognition, temporal ordering, and coordinate and categorical spatial processing. WT mice received intranasal scrambled siRNA (10 $\mu \mathrm{g} /$ mouse) or BDNF siRNA (1, 3, or $10 \mu \mathrm{g} /$ mouse) 48 hours prior to behavioral testing.

(A) Scrambled siRNA-treated WT mice spent significantly more time exploring the novel (N) object than the familiar (F) object (data shows the object exploration time expressed as percent of time exploring each of the objects) $\left(n=11 ; t_{(20)}=7.27,{ }^{*} P<0.01\right)$, and this was dose-dependently impaired by treatment with BDNF siRNA ( $1 \mu \mathrm{g} /$ mouse; $n=9 ; \mathrm{t}_{(16)}=4.47$, ${ }^{*} P<0.01 ; 3 \mu \mathrm{g} /$ mouse: $n=10 ; \mathrm{t}_{(18)}=0.80$, n.s.; $10 \mu \mathrm{g} /$ mouse: $n=13$; $\mathrm{t}_{(24)}=1.14$, n.s.; compared with $\%$ time spent exploring familiar object). (B) Discrimination index is shown for novel object recognition $\left(F_{(3.42)}=6.30, P<0.01\right)\left({ }^{*} P<0.05\right.$ compared with scrambled siRNA-treated mice $[0 \mu \mathrm{g}])$. (C) Treatment with BDNF siRNA impaired coordinate spatial processing $\left(n=9-10 ; \mathrm{F}_{(3,36)}=4.29, P<0.05\right)\left({ }^{*} P<0.05\right.$ compared with scrambled siRNA-treated mice $[0 \mu \mathrm{g}])$, and $(\mathbf{D})$ categorical spatial processing $\left(n=7-10 ; \mathrm{F}_{(3,33)}=7.89, P<0.01\right){ }^{*} P<0.01$ compared with scrambled siRNA-treated mice $[0 \mu \mathrm{g}])$. Values are means $\pm \mathrm{SEM}$. Each symbol represents the value from an individual mouse. $F$ values found by one-way ANOVA. ulate HDACs in the brain and modulate learning and memory. GSK3 knockin mice were tested because they recapitulate the impairments of $\mathrm{Fmrl}^{-/-}$mice in novel object recognition, temporal order memory, and coordinate spatial processing - but not categorical spatial processing - and the cause of the impairments in GSK3 knockin mice is more clearly defined (hyperactive GSK3) compared with a wider range of signaling and morphological alterations that have been reported in $\mathrm{Fmrl}^{\mathrm{1}^{--}}$mice (35). HDAC2 may be the most well-established subtype of HDACs known to regulate learning and memory (15-18). Therefore, we tested if intranasal administration of HDAC2 siRNA is capable of ameliorating impairments in learning and memory in GSK3 knockin mice. Intranasal administration of HDAC2 siRNA, but not scrambled siRNA, improved performance of GSK3 knockin mice in novel object recognition, temporal order memory, and coordinate spatial processing (categorical spatial processing was not examined because it is not impaired in GSK3 knockin mice), but it did not significantly alter the behavior of WT mice (Figure 3). Intranasal HDAC2 siRNA administration was associated with a significant knockdown of hippocampal HDAC2 levels (Supplemental Figure 2). We compared the effects of intranasal administration of HDAC2 siRNA in $\mathrm{Fmrl}^{-/-}$mice to GSK3 mice and found similar improvements in novel object recognition and categorical spatial processing after intranasal HDAC2 siRNA administration in $\mathrm{Fmr1}^{-/-}$mice (Figure 4). However, the 2 models of impaired cognition differed in that, in $\mathrm{Fmrl}^{-/-}$mice, there was only a modest improvement in temporal order memory after HDAC2 siRNA administration and no improvement in coordinate spatial processing (Figure 4), importantly demonstrating that different causes of cognitive deficits affect the outcomes of interventions. Overall, these results demonstrate that intranasal

administration of HDAC2 siRNA is sufficient to reduce HDAC2 levels in the hippocampus and to reverse some of the cognitive deficits in GSK3 knockin mice and $\mathrm{Fmrl}^{-/-}$mice.

We next compared the effects on learning and memory of intranasally administering siRNA targeting HDAC1, HDAC3, or HDAC4 to those of HDAC2 siRNA, which revealed unique actions for each of the 4 HDAC subtypes. Performance of WT mice in novel object recognition, temporal order memory, and coordinate spatial processing were unaffected by intranasal administration of siRNA targeting HDAC1 (Figure 5) or HDAC3 (Figure 6), except for reduced novel object discrimination index by HDAC1 siRNA. In GSK3 knockin mice, intranasal administration of siRNA targeting HDAC1 (Figure 5) improved novel object rec- 
A

\begin{tabular}{l}
\hline Scrambled siRNA or HDAC2 siRNA treatments at arrows \\
\begin{tabular}{|c|c|c|c|c|c|}
\hline & \multicolumn{3}{c}{$\begin{array}{c}\text { Memory } \\
\text { tasks }\end{array}$} \\
\hline $24 \mathrm{~h}$ & $24 \mathrm{~h}$ & $24 \mathrm{~h}$ & $3 \mathrm{~h}$ & $21 \mathrm{~h}$ & $3 \mathrm{~h}$ \\
\hline
\end{tabular}
\end{tabular}

\section{Novel object recognition}

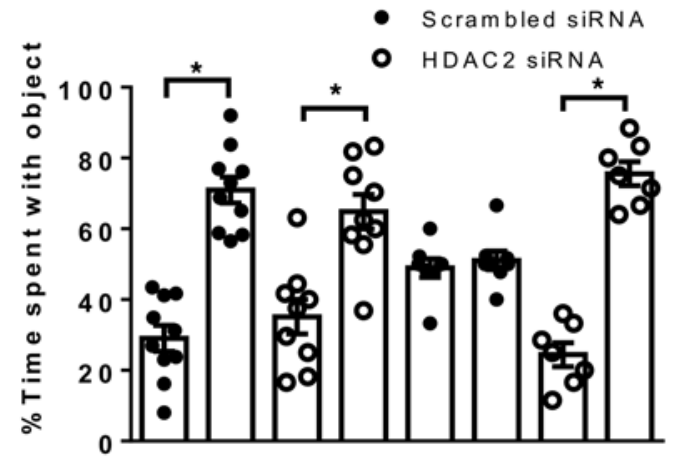

Object: $\quad \frac{F N \text { N N }}{W T} \frac{N \quad F \quad N}{}$

\section{Temporal order recognition}

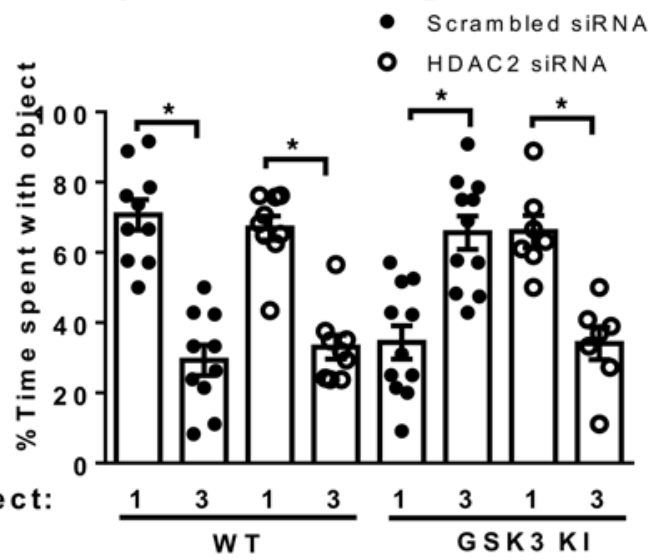

\section{B Novel object recognition}

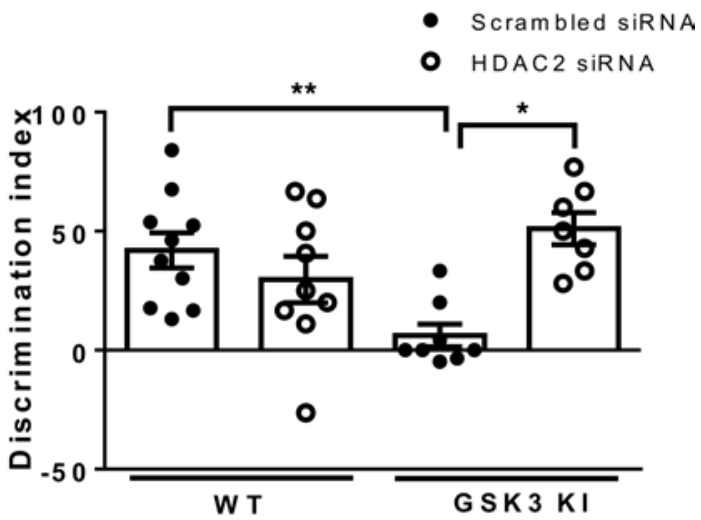

D Temporal order recognition

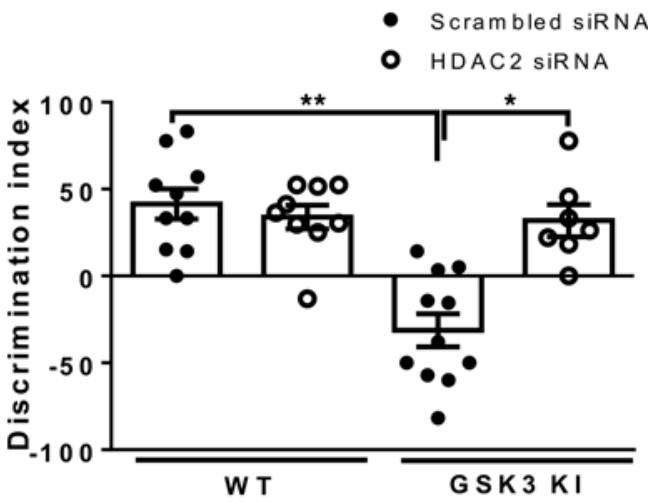

\section{E Coordinate spatial processing}

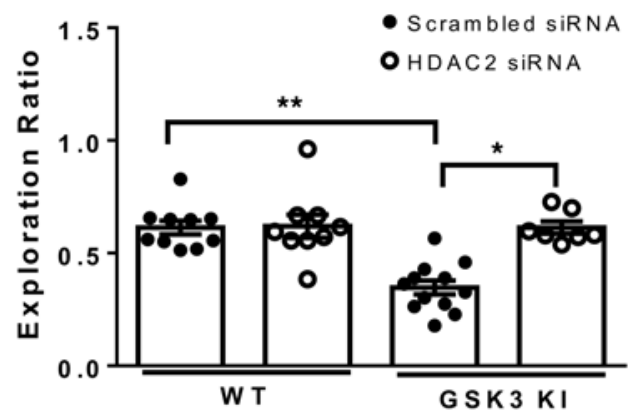

Figure 3. Cognitive performance of WT and glycogen synthase kinase-3 (GSK3) knockin mice after intranasal treatment with histone deacetylase-2 (HDAC2) siRNA. We tested if intranasal administration of siRNA targeting HDAC2 improved novel object recognition, temporal order memory, and coordinate spatial processing that are impaired in GSK3 knockin mice, which recapitulate these impairments of $\mathrm{FmrT}^{-1-}$ mice due to the hyperactive GSK3. Mice were treated intranasally daily with scrambled siRNA or HDAC2 siRNA ( $10 \mu \mathrm{g} / \mathrm{mouse} /$ day) for 3 consecutive days prior to testing and daily throughout the behavioral testing. (A) WT mice spent significantly more time exploring the novel (N) object than the familiar (F) object regardless of treatment (scrambled siRNA: $n=10 ; t_{(18)}=8.02$, ${ }^{*} P<0.01$; HDAC2 siRNA: $n=9$; $\left.\mathrm{t}_{(16)}=4.32,{ }^{*} P<0.01\right)$. GSK3 knockin (KI) mice treated with scrambled siRNA did not display a preference for the novel object $\left(n=8 ; \mathrm{t}_{(14)}=0.58\right.$, n.s.), but this was restored by treatment with HDAC2 siRNA $\left(n=7 ; \mathrm{t}_{(12)}=10.73,{ }^{*} P<0.01\right)$. (B) Discrimination index is shown for novel object recognition (one-way ANOVA: $F_{(3,33)}=5.98, P<0.01$ ) ${ }^{* *} P<0.05$ compared with scrambled siRNA-treated WT mice; ${ }^{*} P<0.01$ compared with scrambled siRNA-treated KI mice). (C) WT mice spent significantly more time exploring the first object presented (1) than the most recent object (3) regardless of treatment (scrambled siRNA: $n=10 ; \mathrm{t}_{(18)}=6.76,{ }^{*} P<$ 0.01 ; HDAC2 siRNA: $n=9 ; \mathrm{t}_{(16)}=7.03,{ }^{*} P<0.01$ ). CSK3 KI mice treated with scrambled siRNA displayed a deficit in temporal ordering performance that was rescued by treatment with HDAC2 siRNA (scrambled siRNA: $n=11 ; \mathrm{t}_{(20)}=4.65,{ }^{*} P<0.01$; HDAC2 siRNA: $n=7 ; \mathrm{t}_{(12)}=4.85$, $\left.{ }^{*} P<0.01\right)$. (D) Discrimination index is shown for temporal ordering ( $n=7-11$; one-way ANOVA: $\left.F_{(3,36)}=16.58, P<0.01\right)\left({ }^{* *} P<0.01\right.$ compared with scrambled siRNA-treated WT mice; ${ }^{*} P<$ 0.01 compared with scrambled siRNA-treated KI mice). (E) Coordinate spatial processing was impaired in GSK3 KI mice but was rescued after HDAC2 siRNA treatment ( $n=7-12$; one-way ANOVA; $\left.F_{(3,37)}=15.66, P<0.01\right)\left({ }^{* *} P<0.01\right.$ compared with scrambled siRNA-treated WT mice; ${ }^{*} P<0.01$ compared with scrambled siRNA-treated $\mathrm{KI}$ mice). Values are means $\pm \mathrm{SEM}$. Each symbol represents the value from an individual mouse. 
A

\begin{tabular}{|c|c|c|c|c|c|}
\hline \multicolumn{3}{|c|}{ Scrambled siRNA or HDAC2 siRNA treatments at arrows } & \multirow{2}{*}{\multicolumn{2}{|c|}{$\begin{array}{c}\text { Memory } \\
\text { tasks }\end{array}$}} & \multirow{2}{*}{$\begin{array}{c}\text { Memory } \\
\text { tasks } \\
h\end{array}$} \\
\hline$\sqrt{2}$ & & & & & \\
\hline $24 \mathrm{~h}$ & $24 \mathrm{~h}$ & $24 \mathrm{~h}$ & 3h & $21 \mathrm{~h}$ & $3 \mathrm{~h}$ \\
\hline
\end{tabular}

Novel object recognition

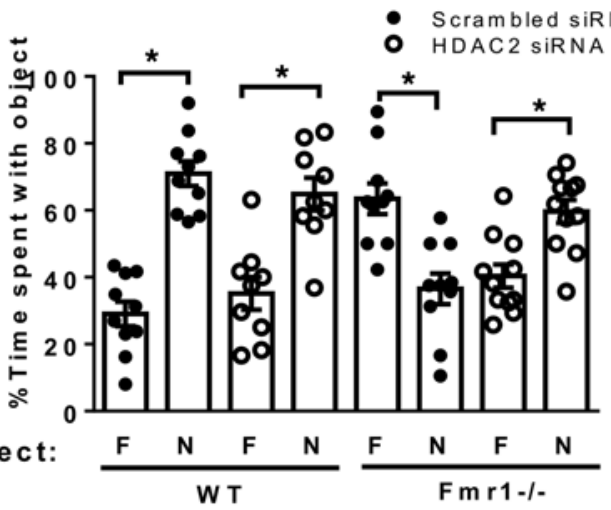

C Temporal order recognition

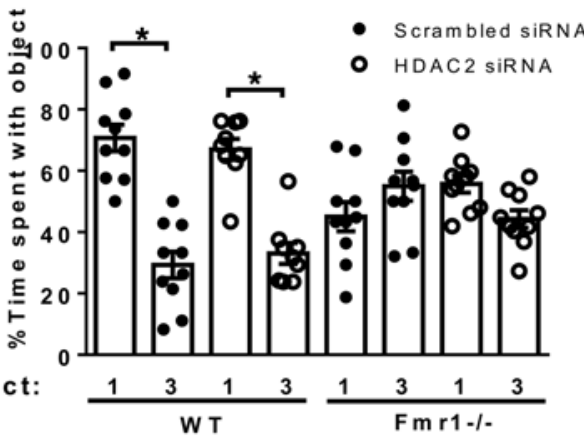

E Coordinate spatial processing

- Scrambled siRna

- hDAC2 sirna

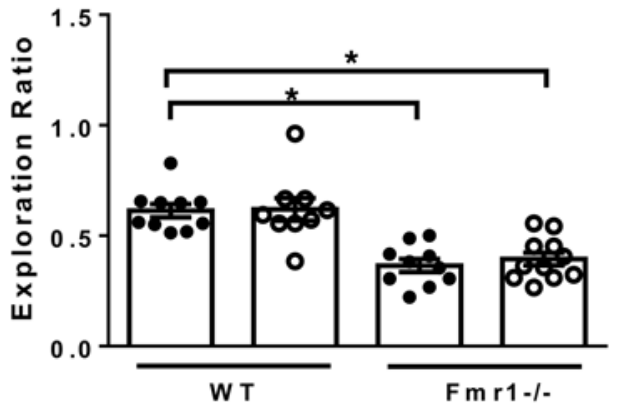

B Novel object recognition

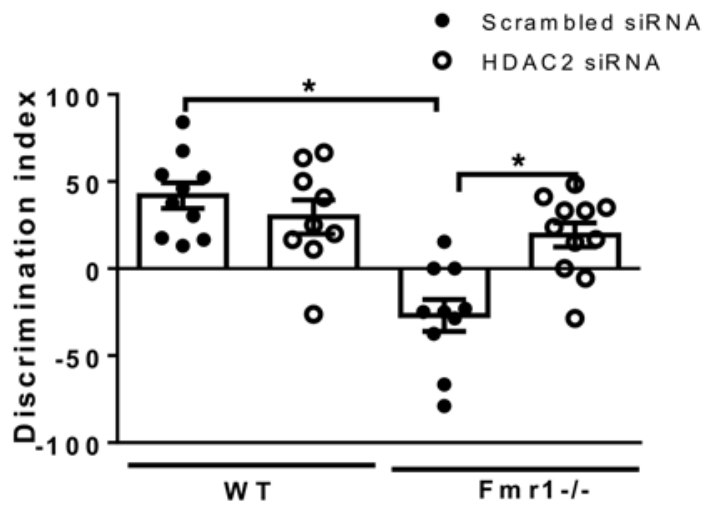

D Temporal order recognition

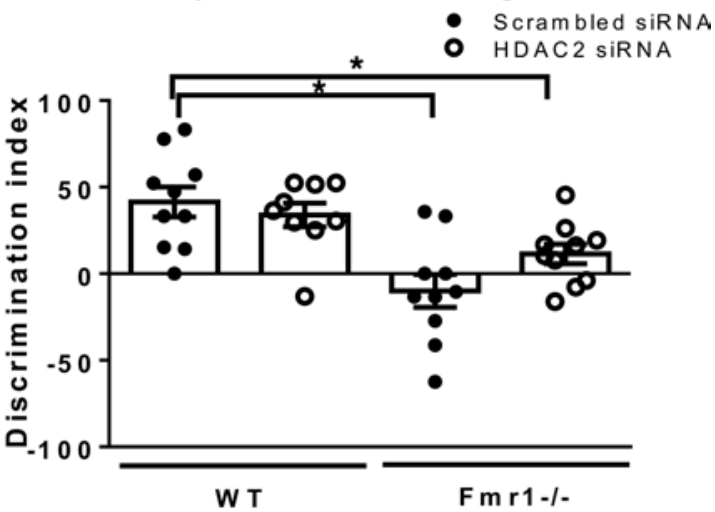

F Categorical spatial processing

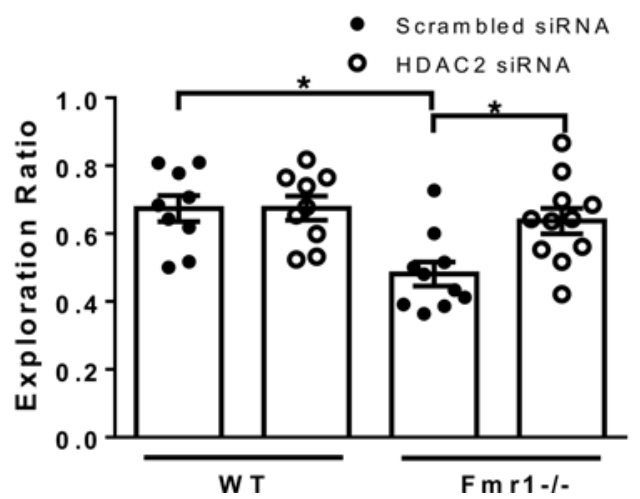

Figure 4. Cognitive performance of WT and $\mathrm{Fmr1}^{-1-}$ mice after intranasal treatment with histone deacetylase-2 (HDAC2) siRNA. We tested if intranasal administration of siRNA targeting HDAC2 improved novel object recognition, temporal order memory, and coordinate spatial processing in Fmr1 ${ }^{-1-}$ mice as it did in GSK3 knockin mice. Mice were treated intranasally daily with scrambled siRNA or HDAC2 siRNA (10 $\mu \mathrm{g} /$ mouse/day) for 3 consecutive days prior to testing and daily throughout the behavioral testing. (A) WT mice spent significantly more time exploring the novel (N) object than the familiar (F) object regardless of treatment (scrambled siRNA: $n=10 ; \mathrm{t}_{(18)}=8.02,{ }^{*} P<0.01$; HDAC2 siRNA: $n=9 ; \mathrm{t}_{(16)}=4.32,{ }^{*} P<0.01$ ). Fmr1 ${ }^{-1-}$ mice treated with scrambled siRNA did not display a preference for the novel object $\left(n=10 ; \mathrm{t}_{(20)}=3.97,{ }^{*} P<0.01\right)$, but this was restored by treatment with HDAC2 siRNA ( $n=11 ; \mathrm{t}_{(18)}=$ 4.13, ${ }^{*} P<0.01$ ). (B) Discrimination index is shown for novel object recognition (one-way ANOVA: $F_{(3,36)}=13.10, P<0.01$ ) (** $P<0.05$ compared with scrambled siRNA-treated WT mice; ${ }^{*} P<0.05$ compared with scrambled siRNA-treated $\mathrm{Fmr1}^{-1-}$ mice). (C) WT mice spent significantly more time exploring the first object presented (1) than the most recent object (3) regardless of treatment (scrambled siRNA: $n=10 ; t_{(18)}=6.76,{ }^{*} P<0.01 ; H D A C 2$ siRNA: $n=9 ; t_{(16)}=7.03$, $\left.{ }^{*} P<0.01\right)$. Fmr1 ${ }^{-1-}$ mice treated with scrambled siRNA displayed a deficit in temporal ordering performance that was partially restored by treatment with 
HDAC2 siRNA without reaching control levels (scrambled siRNA: $n=10 ; \mathrm{t}_{(18)}=2.86, P=0.16 ;$ HDAC2 siRNA: $n=10 ; \mathrm{t}_{(18)}=2.86, P<0.05$ ). (D) Discrimination index is shown for temporal ordering (one-way ANOVA: $\left.F_{(3,35)}=8.79, P<0.01\right)\left({ }^{*} P<0.05\right.$ compared with scrambled siRNA-treated WT mice). (E) Coordinate spatial processing was impaired in $\mathrm{Fmr1}^{-1-}$ mice and was not rescued after HDAC2 siRNA treatment (one-way ANOVA; $\left.n=9-11 ; \mathrm{F}_{(3,36)}=15.33, P<0.01\right)(* * P<$ 0.05 compared with scrambled siRNA-treated WT mice). (F) Categorical spatial processing was impaired in Fmr1/- mice but was rescued after HDAC2 siRNA treatment (one-way ANOVA; $\left.n=9-11 ; F_{(3,35)}=6.22, P<0.01\right)\left({ }^{*} P<0.05\right.$ compared with scrambled siRNA-treated WT mice; ${ }^{*} P<0.05$ compared with scrambled siRNA-treated $\mathrm{Fmrl}^{-1-}$ mice). Values are means \pm SEM. Each symbol represents the value from an individual mouse.

ognition and coordinate spatial processing, but not temporal order memory; HDAC3 siRNA (Figure 6) improved novel object recognition and temporal order memory, but not coordinate spatial processing. In contrast to the effects of targeting the other HDAC subtypes, intranasal HDAC4 siRNA treatment of WT mice impaired novel object recognition, temporal ordering, and coordinate and categorical spatial processing (Figure 7). Immunohistochemical analysis confirmed significant knockdown of hippocampal HDAC1, -3 , and -4 levels after siRNA treatments (Supplemental Figures 3-5). Thus, intranasal administration of siRNAs was sufficient to knockdown hippocampal levels of HDAC subtypes and to reveal subtype-selective modulatory effects of HDACs in several cognitive tasks.

Analysis of targets impairing learning and memory after intranasal administration of BDNF siRNA and HDAC4 siRNA. To identify targets impairing cognitive performance, RNA-seq was carried out on the hippocampi of mice treated intranasally with scrambled siRNA, BDNF siRNA, or HDAC4 siRNA to identify potential mediators of impaired learning and memory caused by knocking down BDNF or HDAC4. Sequence analyses identified several common targets of BDNF siRNA and HDAC4 siRNA administration, and 3 of these were verified by quantitative PCR (qPCR) (Supplemental Figure 6). Among these targets was IGF2, a protein previously reported to enhance memory (36-39).

Intranasal IGF2 treatment improves impaired learning and memory caused by intranasal HDAC4 or BDNF siR$N A$ administration. After knocking down BDNF or HDAC4 with intranasal siRNA administration to induce impairments in learning and memory in WT mice, IGF2 protein was administered intranasally to test if it reversed the impairments. Intranasal treatment with IGF2 reversed cognitive impairments caused by administration of HDAC4 siRNA in the novel object recognition and coordinate and categorical spatial processing (Figure 8) or administration of BDNF siRNA in novel object recognition and coordinate spatial processing (Figure 9). Intranasal IGF2 administration did not alter the performance of WT mice in novel object recognition, temporal ordering, or coordinate and categorical spatial processing (Supplemental Figure 7). Thus, IGF2 is a common target regulated by HDAC4 and BDNF that modulates some aspects of learning and memory.

Hippocampal IGF2 levels and behavior after intranasal treatment with IGF2 and IGF2 siRNA. To further assess the potential cognition-regulating role of IGF2, we tested if changes in IGF2 contribute to learning and memory impairments in $\mathrm{Fmrl}^{\mathrm{I}^{-1}}$ mice or to the beneficial effects of GSK3 $\beta$ siRNA administration. Measurements of the levels of hippocampal IGF2 in $\mathrm{Fmrl}^{-/-}$mice, with or without GSK3 $\beta$ siRNA treatment, revealed that IGF2 levels were lower in the hippocampus of $F m r 1^{-1-}$ mice than WT mice, and GSK3 $\beta$ siRNA treatment increased hippocampal IGF2 levels in $\mathrm{Fmrl}^{-1-}$ mice to that of WT mice (Figure 10A). Furthermore, intranasal IGF2 administration partially ameliorated cognitive impairments in $\mathrm{Fmrl}^{\mathrm{I}^{-/}}$mice in novel object recognition (Figure 10, B and C), temporal ordering (Figure 10, D and E), and coordinate (Figure 10F) and categorical spatial processing (Figure 10G). We also found that intranasal administration of IGF2 siRNA impaired the cognition-repairing effects of the specific GSK3 inhibitor TDZD-8 (5 mg/ kg; i.p.) in $\mathrm{Fmrl}^{-{ }^{--}}$mice in novel object recognition (Figure 11, A and B), temporal ordering (Figure 11, C and $\mathrm{D}$ ), and coordinate (Figure 11E) and categorical spatial processing (Figure 11F). These results demonstrated that IGF2 administration is sufficient to ameliorate cognitive deficits in $\mathrm{Fmrl}^{\mathrm{I}^{--}}$mice and that IGF2 contributes to the beneficial effects of GSK3 inhibitor treatment.

\section{Discussion}

Here, we report that intranasal administration of siRNA can be used to modulate learning and memory in mice. The results show that intranasal administration of siRNAs targeting GSK3 $\beta$ and certain HDAC subtypes can ameliorate existing impairments in learning and memory. Furthermore, intranasal administration of siRNAs can identify proteins required for learning and memory, as siRNAs targeting BDNF and HDAC4 induced impairments.

Previous approaches using siRNA to modulate CNS functions include intraregional and intracerebroventricular administration, which demonstrated that siRNAs possess sufficient stability and transport 
A

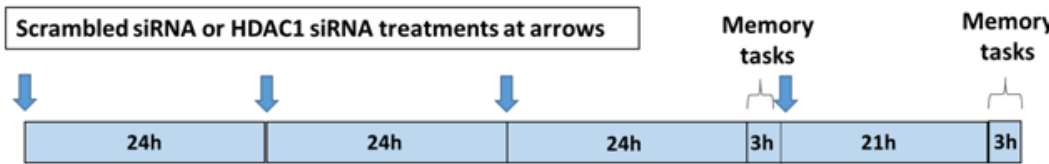

Novel object recognition

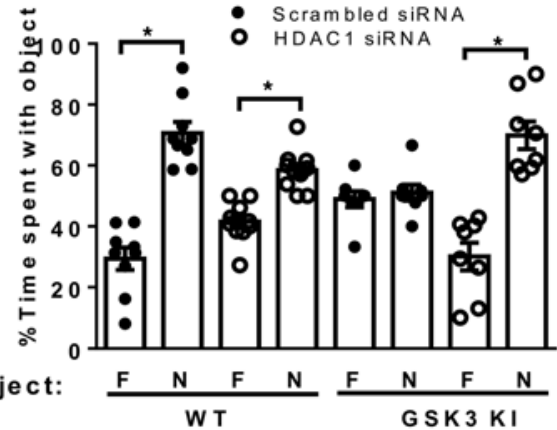

\section{B Novel object recognition}

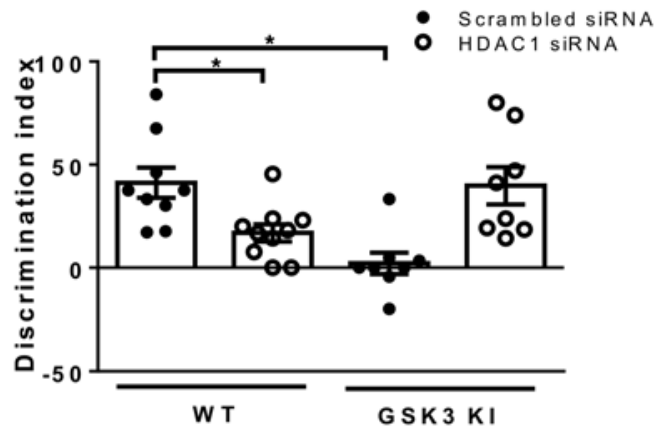

C

Temporal order recognition

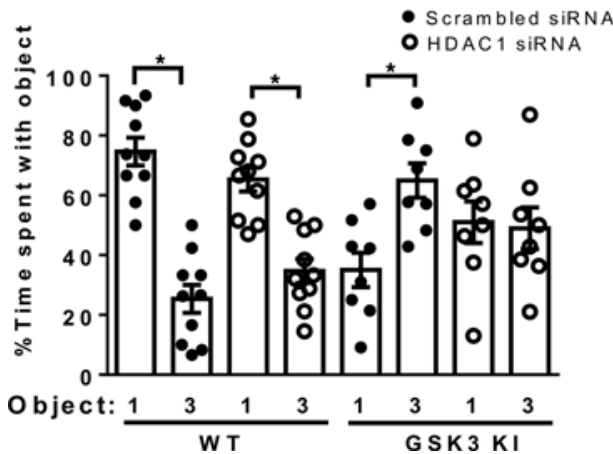

D Temporal order recognition

E Coordinate spatial processing

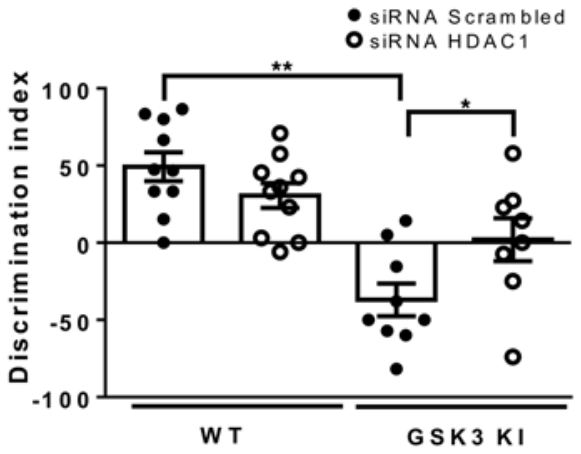

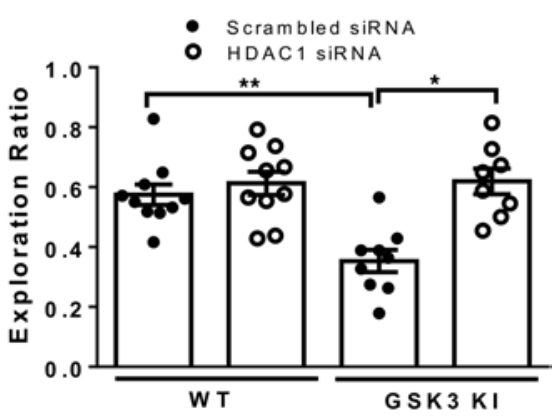

Figure 5. Cognitive performance of WT and glycogen synthase kinase-3 (GSK3) knockin mice after intranasal treatment with histone deacetylase-1 (HDAC1) siRNA. We tested if intranasal administration of siRNA targeting HDAC1 improved novel object recognition, temporal order memory, and coordinate spatial processing that are impaired in CSK3 knockin mice, which recapitulate these impairments of $\mathrm{Fmrl}^{-1-}$ mice due to the hyperactive GSK3. Mice were treated intranasally with scrambled siRNA or HDAC1 siRNA $(10 \mu \mathrm{g} / \mathrm{mouse} /$ day) for 3 consecutive days prior to testing and daily throughout the behavioral testing. (A) WT mice spent significantly more time exploring the novel (N) object than the familiar (F) object regardless of treatment (scrambled siRNA: $n=$ $9 ; \mathrm{t}_{(16)}=7.93,{ }^{*} P<0.01$; HDAC1 siRNA: $n=10 ; \mathrm{t}_{(18)}=5.72,{ }^{*} P<0.01$ compared with $\%$ time spent exploring familiar object). CSK3 knockin (KI) mice treated with scrambled siRNA displayed a deficit in novel object recognition that was rescued by treatment with HDAC1 siRNA (scrambled siRNA: $n=8 ; t_{(14)}=0.58, n .5$.; HDAC1 siRNA: $\left.n=8 ; \mathrm{t}_{(14)}=6.20,{ }^{*} P<0.01\right)$. (B) Discrimination index is shown for novel object recognition (one-way ANOVA: $\left.F_{(3,36)}=10.74, P<0.01\right)(* * P<0.01$ compared with scrambled siRNA-treated WT mice; ${ }^{*} P<0.01$ compared with scrambled siRNA-treated KI mice). (C) WT mice spent significantly more time exploring the first object (1) presented than the most recent object (3) regardless of treatment (scrambled siRNA: $n=10 ; t_{(18)}=7.43,{ }^{*} P<0.01 ; H D A C 1$ siRNA: $\left.n=10 ; \mathrm{t}_{(18)}=5.38,{ }^{*} P<0.01\right)$. CSK3 KI mice treated with scrambled siRNA displayed a deficit in temporal ordering that was significantly altered by treatment with HDAC1 siRNA (scrambled siRNA: $n=9 ; \mathrm{t}_{(14)}=3.67,{ }^{*} P<0.01$; HDAC1 siRNA: $n=8 ; \mathrm{t}_{(14)}=0.20$, n.5. compared with $\%$ time spent exploring first object). (D) Discrimination index is shown for temporal ordering (one-way ANOVA $\left.F_{(3,36)}=13.31, P<0.01\right)\left({ }^{* *} P<0.01\right.$ compared with scrambled siRNA-treated WT mice; ${ }^{*} P<0.01$ compared with scrambled siRNA-treated KI mice). (E) Coordinate spatial processing was impaired in GSK3 KI mice and rescued after HDAC1 siRNA treatment (one-way ANOVA: $\left.\mathrm{F}_{(3,36)}=10.74, n=8-10 ; P<0.01\right)\left({ }^{* *} P<0.01\right.$ compared with scrambled siRNA-treated WT mice; ${ }^{*} P<0.01$ compared with scrambled siRNA-treated $\mathrm{KI}$ mice). Each symbol represents the value from an individual mouse.

properties in the brain to modulate neuronal functions (40-42). However, those approaches require anesthesia and surgery, creating obstacles for translation to clinical applications. Intranasal administration has been used effectively with siRNAs coupled to a variety of carriers, such as nanoparticles, demonstrating the capacity of coupled siRNAs to distribute within the brain after intranasal administration (26-27). Furthermore, functional effects of these intranasally administered carrier-coupled siRNA administration were demonstrated, inducing antidepressant effects (43), diminishing ischemic damage (20), and improving glioma outcome (44). However, we are unaware of any previous reports addressing the capacity of intranasal uncoupled siRNA to affect neuronal functions in vivo, and the present findings demonstrate that siRNA 
A

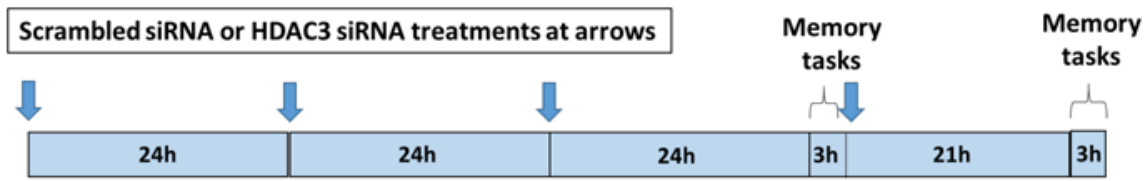

C Temporal order recognition

Novel object recognition

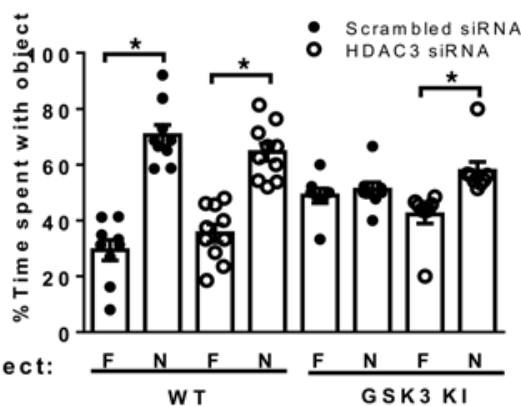

D Temporal order recognition

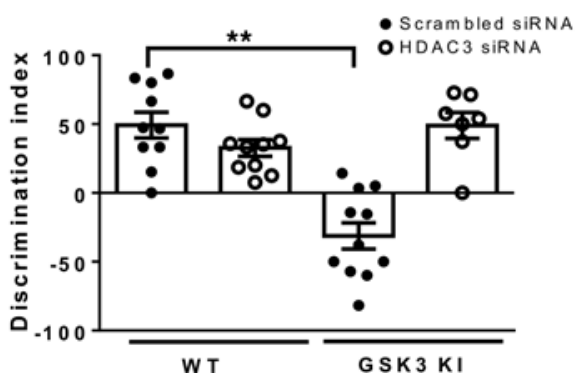

\section{B Novel object recognition}

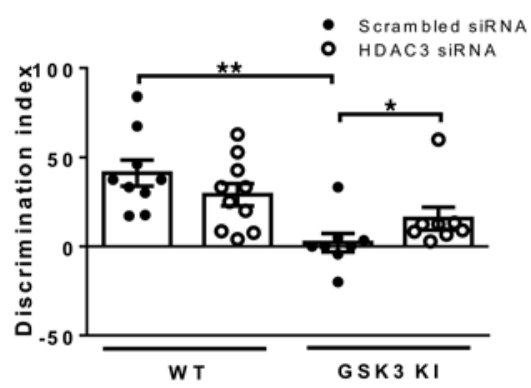

E Coordinate spatial processing

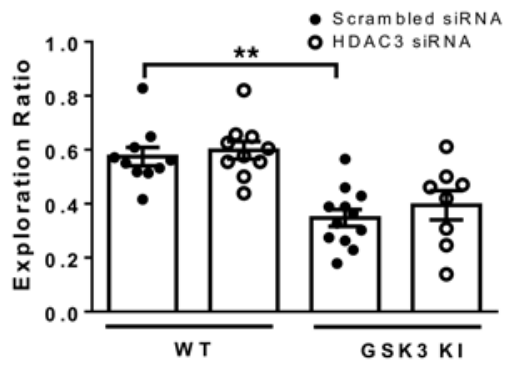

- Scrambled siRna

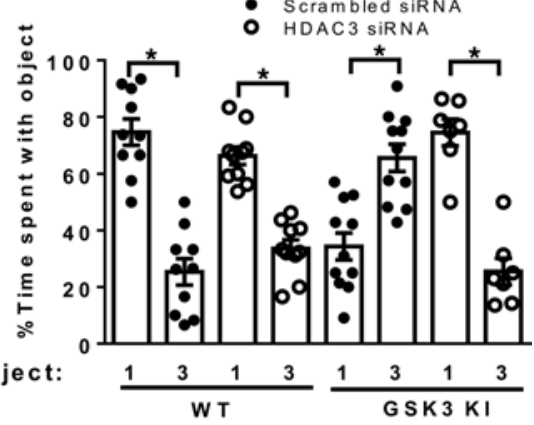

Figure 6. Cognitive performance of WT and glycogen synthase kinase-3 (GSK3) knockin mice after intranasal treatment with histone deacetylase-3 (HDAC3) siRNA. We tested if intranasal administration of siRNA targeting HDAC3 improved novel object recognition, temporal order memory, and coordinate spatial processing that are impaired in GSK3 knockin (KI) mice, which recapitulate these impairments of $\mathrm{Fmr1}^{-1-}$ mice due to the hyperactive CSK3. Mice were treated intranasally with scrambled siRNA or HDAC3 siRNA ( $10 \mu \mathrm{g} / \mathrm{mouse} /$ day) for 3 consecutive days prior to testing and daily throughout the behavioral testing. (A) WT mice spent significantly more time exploring the novel (N) object than the familiar (F) object regardless of treatment (scrambled siRNA: $n=9 ; t_{(16)}=7.94{ }^{*} P<0.01$; HDAC3 siRNA: $n=10 ; t_{(18)}=6.55,{ }^{*} P<0.01$ compared with $\%$ time spent exploring familiar object). GSK3 KI mice treated with scrambled siRNA displayed a deficit in novel object recognition that was improved by treatment with HDAC3 siRNA (scrambled siRNA: $n=8$; $\mathrm{t}_{(14)}=0.58$, n.s.; HDAC3 siRNA: $n=8 ; \mathrm{t}_{(14)}=3.43,{ }^{*} P<0.01$ compared with $\%$ time spent exploring familiar object). (B) Discrimination index is shown for novel object recognition (one-way ANOVA: $F_{(3,34)}=6.65, P<0.01$ ) ${ }^{* *} P<0.01$ compared with scrambled siRNA-treated WT mice). (C) WT mice spent significantly more time exploring the first object (1) presented than the most recent object (3) regardless of treatment (scrambled siRNA: $n=10 ; \mathrm{t}_{(18)}=7.43$, ${ }^{*} P<0.01$; HDAC3 siRNA: $n=10 ; \mathrm{t}_{(18)}=7.59,{ }^{*} P<0.01$ compared with \% time spent exploring the first object). GSK3 KI mice treated with scrambled siRNA displayed a deficit in temporal ordering performance that was rescued by treatment with HDAC3 siRNA (scrambled siRNA: $n=11 ; \mathrm{t}_{(20)}=4.65,{ }^{*} P<0.01$; HDAC3 siRNA: $n=7 ; \mathrm{t}_{(12)}=7.40,{ }^{*} P<0.01$ compared with \% time spent exploring first object). (D) Discrimination index is shown for temporal ordering (one-way ANOVA; $\left.\mathrm{F}_{(3,37)}=20.42 ; P<0.01\right)\left({ }^{*} P<0.01\right.$ compared with scrambled siRNA-treated WT mice; ${ }^{*} P<0.01$ compared with scrambled siRNA-treated KI mice). (E) Coordinate spatial processing was impaired in GSK3 KI mice, and this was not altered by HDAC3 siRNA treatment (one-way ANOVA: $\left.n=8-12 ; F_{(3,39)}=12.16, P<0.01\right)(* * P<0.01$ compared with scrambled siRNA-treated WT mice). Values are means $\pm \mathrm{SEM}$. Each symbol represents the value from an individual mouse.

need not be coupled to a carrier to functionally improve or impair learning and memory. Although questions remain about the mechanisms of siRNA uptake, transport, and stability for each of these approaches, it is evident that siRNA administered intranasally reaches the hippocampus and is sufficient to knock down levels of hippocampal proteins and to modify functions, such as learning and memory. A number of questions remain to be addressed, including a more complete evaluation of the distribution of intranasally administered siRNA, the duration of effects, and potential detrimental effects on cell viability and inflammation of intranasal siRNA administration. Nonetheless, these results demonstrate that intranasal siRNA administration has the potential to supplement other methods to identify mechanisms regulating learning and memory, and possibly other CNS functions, and to ameliorate impairments.

In the mouse model of Fragile X syndrome, peripheral administration of GSK 3 inhibitors reverses deficits in several learning and memory tasks $(6,30,31)$. Avoiding peripheral effects of systemically administered GSK3 inhibitors, we found that intranasal administration of the GSK3 peptide inhibitor 
A

\begin{tabular}{|c|c|c|c|c|c|}
\hline Scrambled siRNA or HDAC4 siRNA treatments at arrows & \multicolumn{3}{c}{$\begin{array}{c}\text { Memory } \\
\text { tasks }\end{array}$} \\
\hline $24 \mathrm{~h}$ & $24 \mathrm{~h}$ & $24 \mathrm{~h}$ & $3 \mathrm{~h}$ & $21 \mathrm{~h}$ & $3 \mathrm{~h}$ \\
\hline
\end{tabular}

Novel object recognition

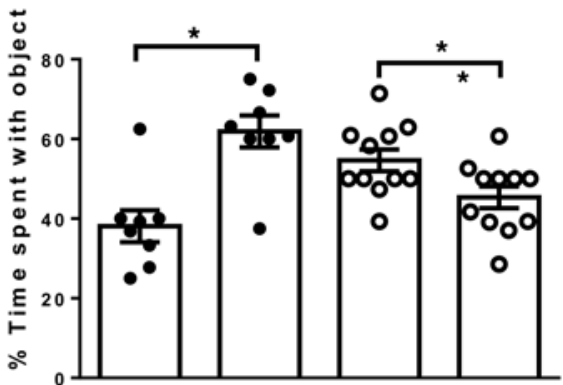

Object:

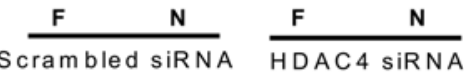

C Temporal order recognition

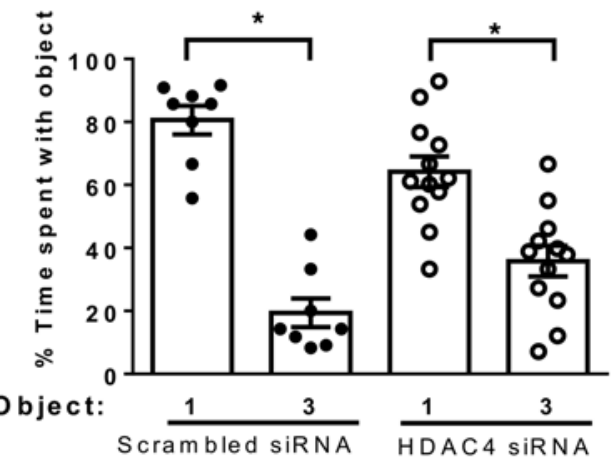

E Coordinate spatial processing

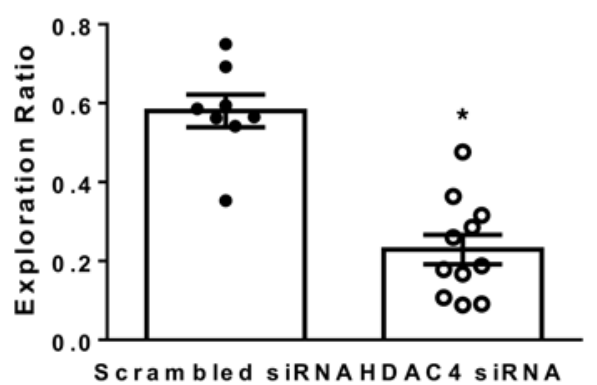

B Novel object recognition

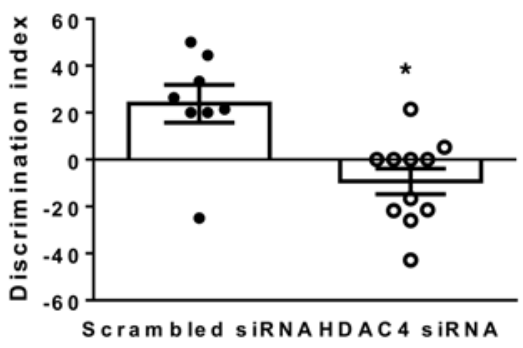

D Temporal order recognition

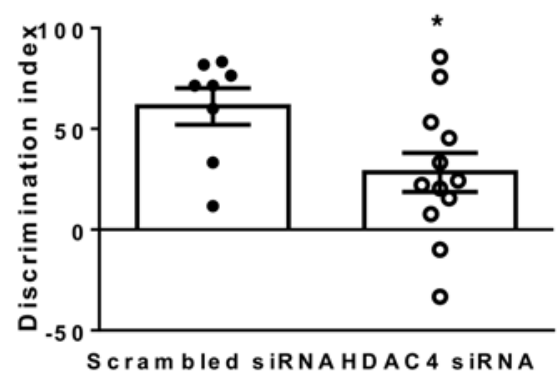

F Categorical spatial processing

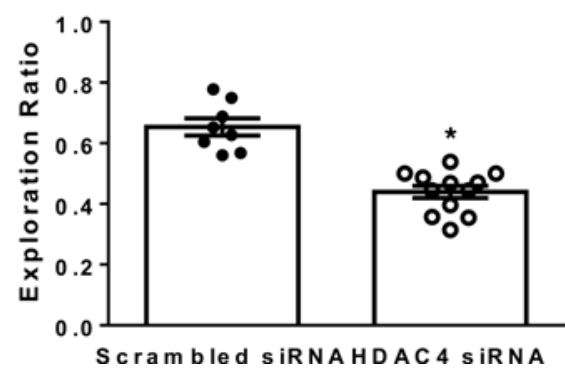

Figure 7. Cognitive performance of WT mice after intranasal treatment with siRNA targeting histone deacetylase-4 (HDAC4). We tested if intranasal administration of siRNA targeting HDAC4 altered novel object recognition, temporal order memory, and coordinate and categorical spatial processing in WT mice. WT mice were treated intranasally daily with scrambled siRNA or siRNA targeting HDAC4 ( $10 \mu \mathrm{g} /$ mouse/day) for 3 consecutive days prior to testing and daily throughout the behavioral testing. (A) WT mice treated with HDAC4 siRNA displayed a deficit in novel object recognition, spending significantly less time exploring the novel $(\mathrm{N})$ object than the familiar $(\mathrm{F})$ object (scrambled siRNA: $n=8$; $\mathrm{t}_{(14)}=$ 4.19, ${ }^{*} P<0.01$; HDAC4 siRNA: $n=11$; $\mathrm{t}_{(20)}=2.41,{ }^{*} P<0.01$ ). (B) Discrimination index is shown for novel object recognition $\left(t_{(17)}=3.54 ;{ }^{*} P<0.01\right.$ compared with scrambled siRNAtreated mice). (C) WT mice spent significantly more time exploring the first object (1) presented than the most recent object (3) regardless of treatment (scrambled siRNA: $n=8$; $\mathrm{t}_{(14)}=9.55,{ }^{*} P<0.01$; HDAC4 siRNA: $\left.n=12 ; \mathrm{t}_{(22)}=4.13,{ }^{*} P<0.01\right)$. (D) Discrimination index is shown for temporal ordering $\left(\mathrm{t}_{(18)}=2.34 ;{ }^{*} P<0.05\right)$. (E) Coordinate spatial processing was impaired in WT mice after HDAC4 siRNA treatment $\left(n=8-11 ; \mathrm{t}_{(17)}=6.26\right.$, $\left.{ }^{*} P<0.01\right)$. (F) Categorical spatial processing was impaired in WT mice after HDAC4 siRNA treatment $(n=$ $\left.8-12 ; \mathrm{t}_{(15)}=5.50,{ }^{*} P<0.01\right)$. Values are means \pm SEM. Each symbol represents the value from an individual mouse. Student's $t$ test was used to analyze each behavioral test.

L803-mts improved learning and memory in $\mathrm{Fmrl}^{-/-}$mice, demonstrating that brain regions relevant to these learning and memory tasks can be accessed intranasally. Remarkably, knockdown of GSK3 $\beta$ by intranasal siRNA administration also was sufficient to improve cognitive performances of $\mathrm{Fmrl}^{-\mathrm{I}^{--}}$mice. Notably, intranasal L803-mts or GSK3 $\beta$ siRNA nearly normalized several severe learning and memory impairments in $\mathrm{Fmrl}^{-1-}$ mice to the level of WT mice. L803-mts inhibits both GSK3 $\beta$ and GSK3 $\alpha$, and since GSK3 $\alpha$ levels were not measured, we cannot exclude the possibility that it may have been knocked down to some extent by GSK3 $\beta$ siRNA and could have contributed to the behavioral effects. Overall, these results demonstrate that both GSK3 inhibitors and siRNA are viable candidates for intranasal interventions to ameliorate impairments in learning and memory.

Finding that intranasal administration of GSK3 $\beta$ siRNA effectively ameliorated impaired learning and memory in $\mathrm{Fmrl}^{-/-}$mice led us to examine if this approach could be used to identify molecules 
A
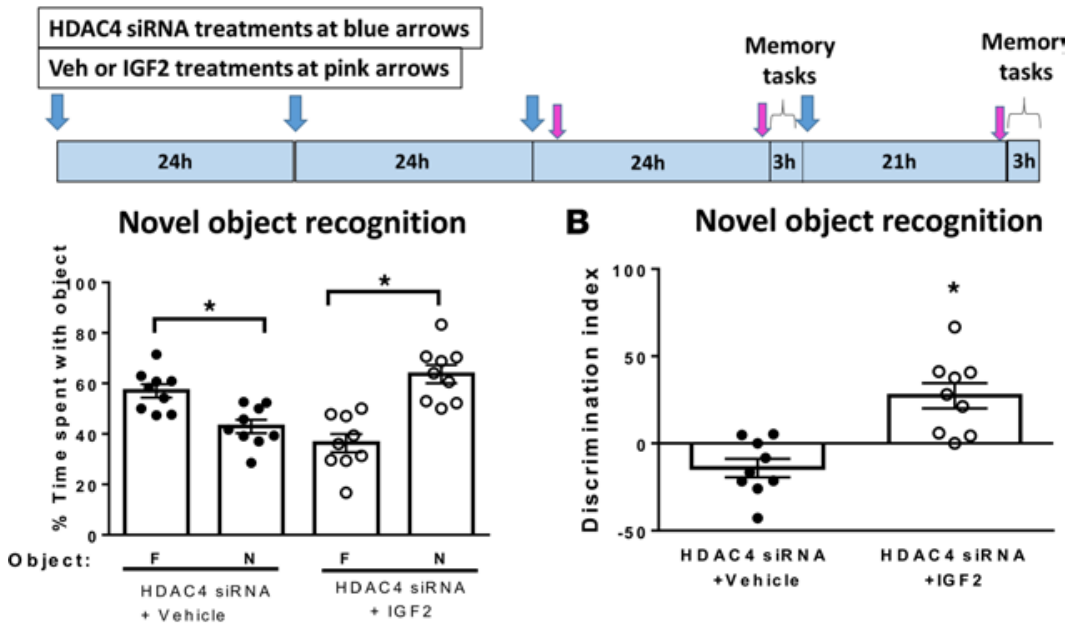

C Temporal order recognition

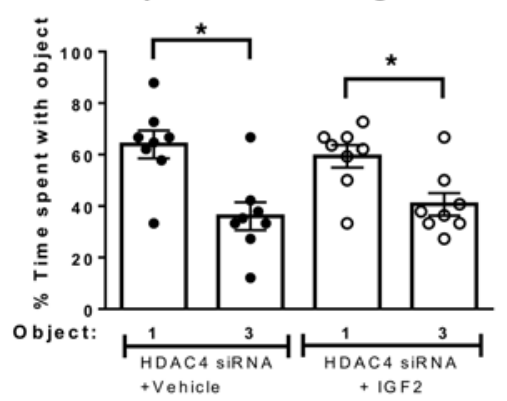

E Coordinate spatial processing

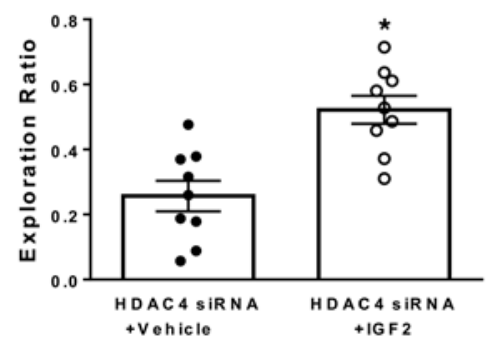

B Novel object recognition

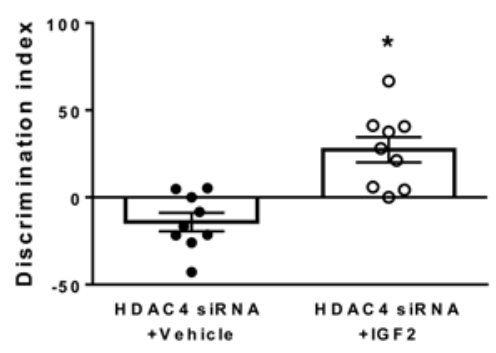

D Temporal order recognition

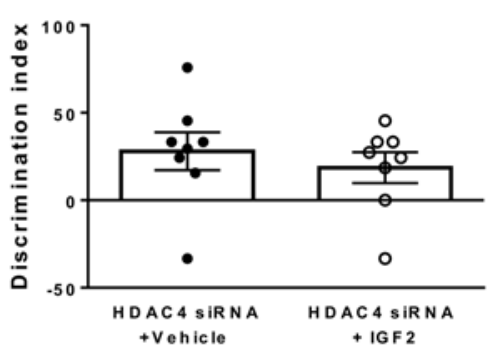

F Categorical spatial processing

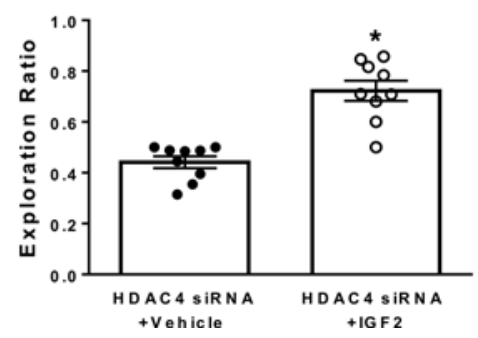

Figure 8. Intranasal treatment with insulin-like growth factor-2 (IGF2) improves impaired cognition caused by intranasal histone deacetylase-4 (HDAC4) siRNA administration. We tested if intranasal administration of IGF2 altered the performance of HDAC4 siRNA-treated WT mice in novel object recognition, temporal order memory, and coordinate and categorical spatial processing to determine if deficient IGF2 contributed to impairments induced by knocking down HDAC4. WT mice were treated intranasally with HDAC4 siRNA ( $10 \mu \mathrm{g} /$ mouse/day) for 3 days followed by vehicle or IGF2 ( $0.1 \mu \mathrm{g} /$ mouse/day) 24 hours and 1 hour prior to behavioral testing. (A) HDAC4 siRNA plus vehicle-treated mice displayed a deficit in novel object recognition that was restored by treatment with IGF2 (vehicle: $n=9$; $\mathrm{t}_{(16)}=3.74,{ }^{*} P<0.01$; IGF2: $n=9$; $\mathrm{t}_{(16)}=5.31,{ }^{*} P<0.01$ ). (B) Discrimination index is shown for novel object recognition $\left(t_{(16)}=4.59,{ }^{*} P<0.01\right)$. (C) Mice treated with HDAC4 siRNA plus vehicle spent significantly more time exploring the first object presented (1) than the most recent object (3) $\left(n=8 ; \mathrm{t}_{(14)}=3.65,{ }^{*} P<\right.$ $0.01)$, and ICF2 treatment did not alter temporal ordering performance $\left(n=8 ; \mathrm{t}_{(14)}=3.00,{ }^{*} P<0.01\right)$. (D) Discrimination index in temporal ordering was not different between vehicle- and IGF2-treated mice $\left(t_{(14)}=0.67\right.$, n.s.). (E) Mice treated with HDAC4 siRNA plus vehicle $(n=9)$ displayed impaired coordinate spatial processing that was improved by treatment with IGF2 $\left(n=9 ; \mathrm{t}_{(16)}=4.16,{ }^{*} P\right.$ $<0.01)$. (F) Mice treated with HDAC4 siRNA plus vehicle $(n=9)$ displayed impaired categorical spatial processing that was restored by treatment with IGF2 $\left(n=9 ; \mathrm{t}_{(16)}=\right.$ $6.14,{ }^{*} P<0.01$ ). Values are means \pm SEM. Each symbol represents the value from an individual mouse. Student's $t$ test was used to analyze each behavioral test.

that regulate learning and memory. Taking advantage of the known requirement for BDNF for some aspects of learning and memory $(45,46)$, we tested if intranasally administered siRNA could be used to identify proteins required for learning and memory. These experiments showed that BDNF siRNA dose-dependently confirmed its requirement for novel object recognition and spatial memory, demonstrating the utility of this approach. Thus, intranasal siRNA administration can be applied to identify targets that are necessary for learning and memory, as well as targets to improve impaired learning and memory.

Finding that the intranasal siRNA approach can be used to determine the modulatory effects of proteins on learning and memory, we applied it to test the involvement of 4 HDAC subtypes in the regulation of learning and memory. HDACs have been classified in 5 main subtypes, and although they all mediate deacetylation, differential effects of HDAC subtypes on learning and memory have been identified, as noted in the Introduction. Intranasal administration of siRNAs targeting HDAC1, -2 , or -3 had minimal effects on learning and memory in WT mice but counteracted impairments displayed by GSK3 knockin mice. In contrast, intranasal HDAC4 siRNA significantly impaired learning and memory in WT mice. These findings demonstrate that intranasal siRNA administration is useful to identify actions of related proteins for which specific inhibitors are lacking, and they demonstrate the functional effects of these 4 HDAC subtypes in modulating learning and memory.

We examined if intranasal BDNF siRNA and HDAC4 siRNA, each of which impaired learning and memory, might affect common targets, which identified IGF2, among others. IGF2 was particularly interesting because a significant body of evidence has demonstrated that it promotes learning and memory (36, 47). Surprisingly, considering the number of targets regulated by BDNF and HDAC4 deficiency, treatment with intranasal IGF2 significantly ameliorated some of the learning and memory deficits induced by 
A

\begin{tabular}{|c|c|}
\hline BDNF siRNA treatment at blue arrow \\
\hline Veh or IGF2 treatments at pink arrows \\
\hline $24 \mathrm{~h}$ & $24 \mathrm{~h}$ \\
\hline
\end{tabular}

Novel object recognition

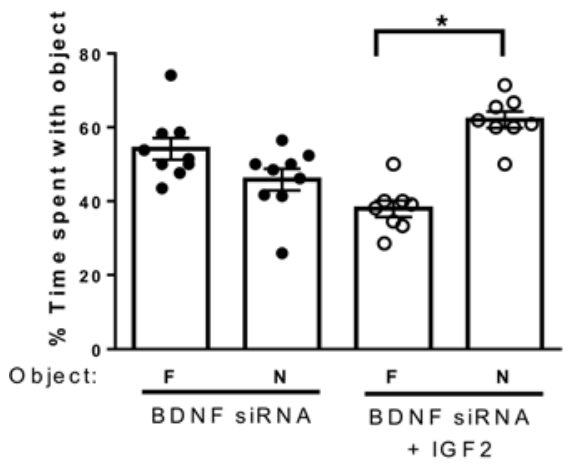

B Novel object recognition

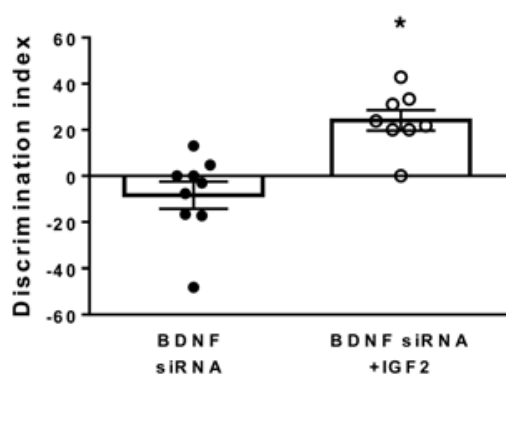

C Coordinate spatial processing

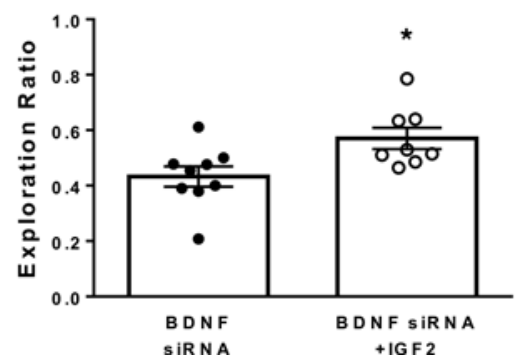

Figure 9. Intranasal treatment with insulin-like growth factor-2 (IGF2) restores impaired cognition after intranasal brain-derived neurotrophic factor (BDNF) siRNA administration. We tested if intranasal administration of IGF2 altered the performance of BDNF siRNA-treated WT mice in novel object recognition and coordinate spatial processing to determine if deficient IGF2 contributed to impairments induced by knocking down BDNF. WT mice received BDNF siRNA ( $3 \mu \mathrm{g}$ / mouse) 48 hours prior to behavioral testing. IGF2 ( $0.1 \mu \mathrm{g} / \mathrm{mouse} /$ day; gray bars) or vehicle (white bars) was administered 24 hours and 1 hour prior to behavioral testing. (A) BDNF siRNA-treated mice displayed a deficit in novel object recognition, displaying no difference in exploration of the novel and familiar objects $\left(n=9 ; t_{(10)}=0.92\right.$, n.s. $)$ that was rescued by treatment with IGF2 and led to preferential exploration of the novel object $(n=8$ $t_{(14)}=7.67,{ }^{*} P<0.01$ compared with \% time spent exploring familiar [F] object). (B) Discrimination index is shown for novel object recognition $\left(t_{(15)}=\right.$ $4.29,{ }^{*} P<0.01$ compared with BDNF siRNA-treated mice). (C) BDNF siRNA-treated mice displayed impairments in coordinate spatial processing $(n=$ 9) that was rescued by treatment with IGF2 $(n=8$; $\mathrm{t}_{(15)}=2.59,{ }^{*} P<0.05$ compared with BDNF siRNAtreated mice). Values are means \pm SEM. Each symbol represents the value from an individual mouse. Student's $t$ test was used to analyze each behavioral test.

BDNF and HDAC4 siRNA administration. These results led to the finding that IGF2 is deficient in the hippocampus of $\mathrm{Fmrl}^{-1-}$ mice and that intranasal IGF2 administration improved learning and memory in $\mathrm{Fmrl}^{-{ }^{-}-}$mice. IGF2 has not previously been linked to intellectual disability in Fragile X syndrome. However, transgenic IGF2-overexpressing $\mathrm{Fmrl}^{-1-}$ mice were reported to display increased audiogenic seizure susceptibility, although it is not known if this was affected by developmental effects of overexpressed IGF2 (48). Altogether, our findings raise the possibility that IGF2 may offer therapeutic benefits in Fragile $\mathrm{X}$ syndrome. This proposal is further supported by the finding that increases in IGF2 contribute to the amelioration of learning and memory deficits attained by inhibition of GSK3 in $\mathrm{Fmrl}^{-/-}$mice.

In summary, these results show that intranasal administration of selected siRNAs is able to effectively identify targets that regulate learning and memory and alleviate or induce impaired learning and memory in mouse models. This approach identified IGF2 as a therapeutic target for rescuing intellectual disability in Fragile X syndrome. Moreover, intranasally administered siRNA may effectively modulate other CNS functions, in addition to modifying targets that can ameliorate or impair cognitive functions in mice.

\section{Methods}

\section{Mice and Drugs}

Male C57BL/ 6 littermates (8-10 weeks old), with or without a disruption of the Fmr1 gene, were used. The $\mathrm{Fmrl}^{-/-}$mice (originally provided by William T. Greenough, University of Illinois, Urbana, Illinois, USA) were generated by breeding male C57BL/6 hemizygous Fmr1 KO mice and female C57BL/6 heterozygous Fmr1 KO mice to generate male homozygous Fmr1 KO mice and WT littermates. Genotypes were determined by polymerase chain reaction using the Jackson Laboratory protocol for genotyping $\mathrm{Fmrl}^{-1-}$ mice. Male adult (8-10 weeks old) homozygous GSK3 $\alpha / \beta^{21 \mathrm{~A} / 21 \mathrm{~A} / 9 \mathrm{~A} / 9 \mathrm{~A}}$ knockin mice and matched WT mice (originally generously provided by Dario Alessi, University of Dundee, Dundee, United Kingdom, ref. 3) were used when indicated. 
A
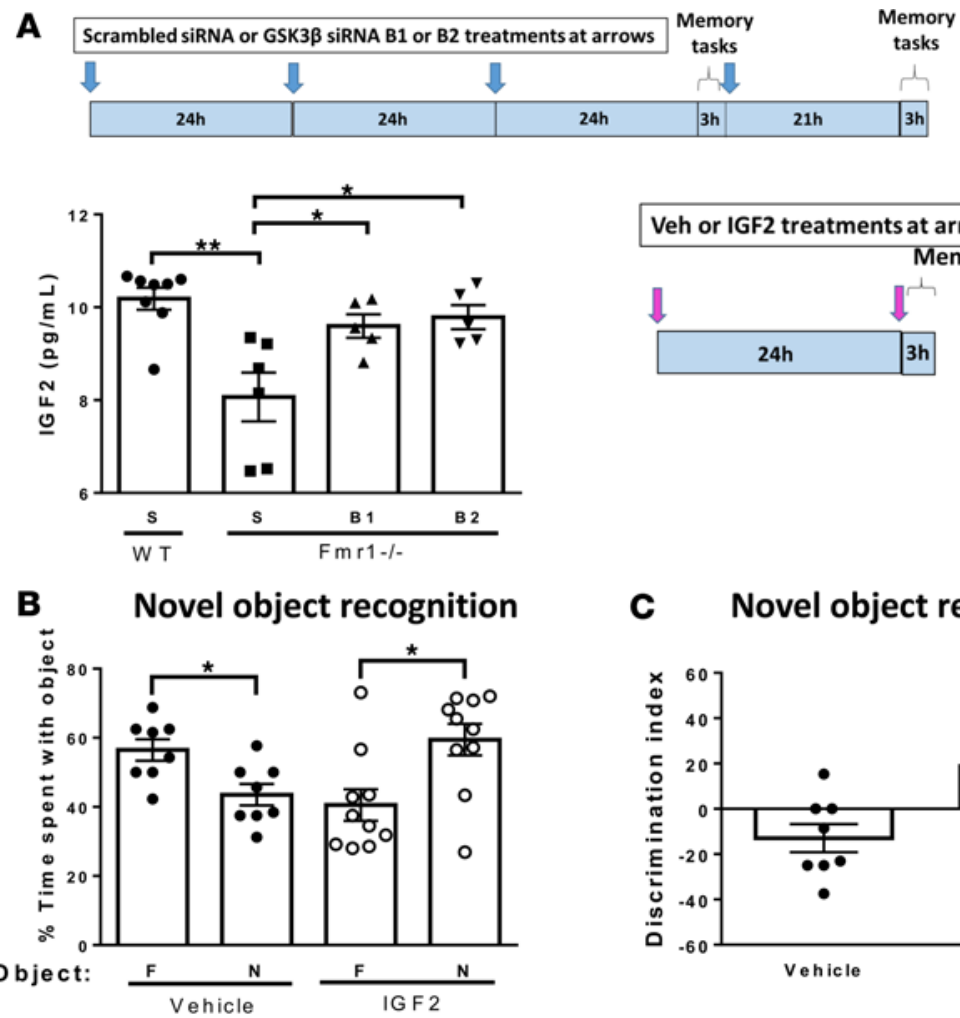

D Temporal order recognition

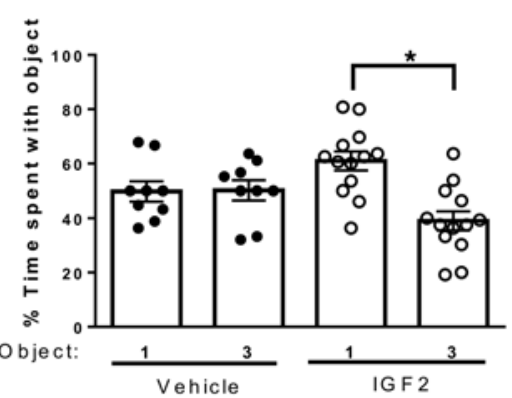

F Coordinate spatial processing

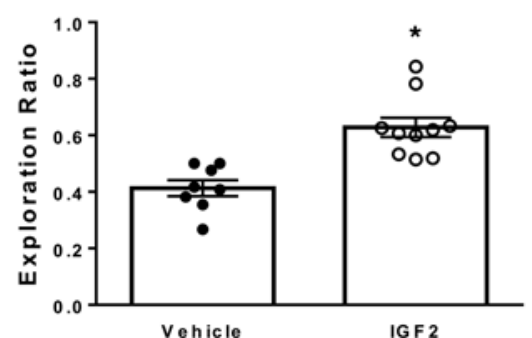

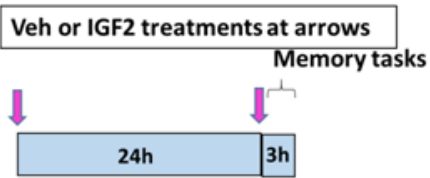

C Novel object recognition

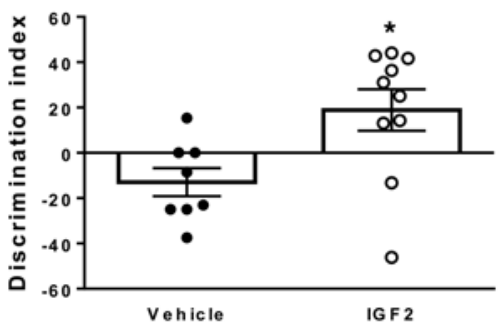

E Temporal order recognition

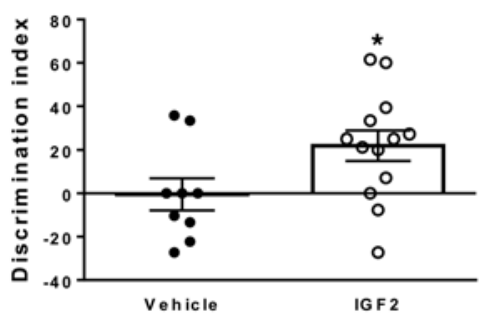

G Categorical spatial processing

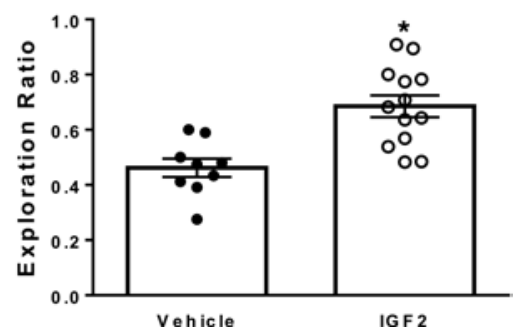

Figure 10. Hippocampal insulin-like growth factor-2 (IGF2) levels and behavior after intranasal treatment with IGF2. We measured hippocampal IGF2 levels to determine if they were lower in $\mathrm{Fmr1}^{-1-}$ mice than in WT mice and to test if knocking down GSK3 $\beta$ increased IGF2 levels in $\mathrm{Fmr1}^{-1-}$ mice. We also tested if intranasal IGF2 administration was sufficient to ameliorate cognitive impairments in $\mathrm{Fmr1}^{-/-}$mice in novel object recognition, temporal ordering, and coordinate and categorical spatial processing. (A) Hippocampal ICF2 levels were measured after behavioral testing and were lower in $\mathrm{Fmr1}^{-1-}$ mice $(n=6)$ than WT mice $(n=8)$ after 4 days of treatment with intranasal scrambled siRNA (S) and were increased in $\mathrm{Fmr}^{-/-}$mice after 4 days of treatment with either of 2 different sequences of GSK3 $\beta$ siRNA B1 (sequence $1 ; n$ = 5), or B2 (sequence 2; $n=5$ ) $10 \mu \mathrm{g} /$ mouse/ day (one-way ANOVA: $F_{(3.20)}=7.84, P<0.01$ ) $\left({ }^{*} P<0.01\right.$ compared with scrambled siRNAtreated WT mice; ${ }^{*} P<0.05$ compared with scrambled siRNA-treated $\mathrm{Fmr1}^{-1-}$ mice). $n=24$ mice. (B-C) $\mathrm{Frmr1}^{-1-}$ mice received intranasal vehicle or IGF2 (0.1 $\mu \mathrm{g} /$ mouse/day) 24 hours and 1 hour prior to behavioral testing. (B) $\mathrm{Fmrr}^{-1-}$ mice treated with vehicle displayed a deficit in novel object recognition that was restored by treatment with IGF2 (vehicle: $n$ $=8 ; \mathrm{t}_{(14)}=2.96,{ }^{*} P<0.05 ;$ IGF2: $n=10 ; \mathrm{t}_{(18)}=$ 2.92, ${ }^{*} P<0.01$ ). (C) Discrimination index is shown for novel object recognition $\left(t_{(15)}=2.73\right.$, $\left.{ }^{*} P<0.05\right)$. (D) $F \mathrm{mr}^{-1-}$ mice treated with vehicle displayed a deficit in temporal ordering that was improved by treatment with IGF2 (vehicle: $n=9$; $\mathrm{t}_{(16)}=0.09$, n.s.; IGF2: $n=13$; $\mathrm{t}_{(12)}$ $\left.=2.22,{ }^{*} P<0.05\right)$. 1 , first object presented; 3 , most recent object presented. (E) Discrimination index is shown for temporal ordering $\left(t_{(14)}=2.16,{ }^{*} P<0.05\right)$. (F) Coordinate spatial processing was impaired in $\mathrm{Fmrr}^{-/-}$mice $(n=$ 8) and restored after IGF2 treatment $(n=10$; $\left.t_{(15)}=4.67,{ }^{*} P<0.05\right)$. (C) Categorical spatial processing was impaired in $\mathrm{Fmrr}^{-1-}$ mice $(n=$ 9) and rescued after IGF2 treatment $(n=13$; $\left.t_{(14)}=3.82,{ }^{*} P<0.05\right)$. Values are means \pm SEM. Each symbol represents the value from an individual mouse.

GSK3 knockin mice develop and reproduce normally with no overt phenotype (3, 49, 50). Male C57BL/6 WT mice (8-10 weeks old) were obtained from Charles River Laboratories. Mice were group-housed in lightand temperature-controlled rooms.

L803-mts, a substrate-competitive peptide specific GSK3 inhibitor, was synthesized in the Eldar-Finkelman laboratory (Tel Aviv University, Tel Aviv, Israel) as previously described (51) and was administered in DDX vehicle (128 mM NaCl, $8 \mathrm{mM}$ citric acid monohydrate, $17 \mathrm{mM}$ disodium phosphate dehydrate, $0.0005 \%$ benzalkonium chloride). L803-mts ( $60 \mu \mathrm{g} / \mathrm{mouse} /$ day) or GSK3 $\beta$ siRNA (10 $\mu \mathrm{g} / \mathrm{mouse} /$ day; D-041080-02 and D-041080-03; GE Healthcare Dharmacon Inc.) were administered intranasally in each nostril ( $5 \mu 1 /$ nostril) for 3 days prior to behavioral testing and daily throughout the behavioral testing. Sequences of all siRNAs are shown in Supplemental Table 1. Thiadiazolidindione-8 (TDZD-8) was 
A

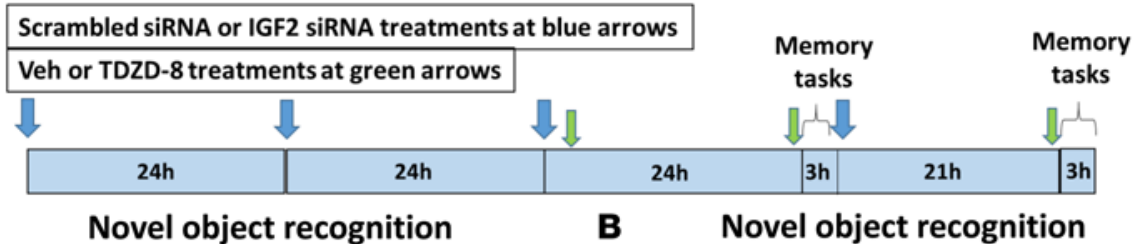

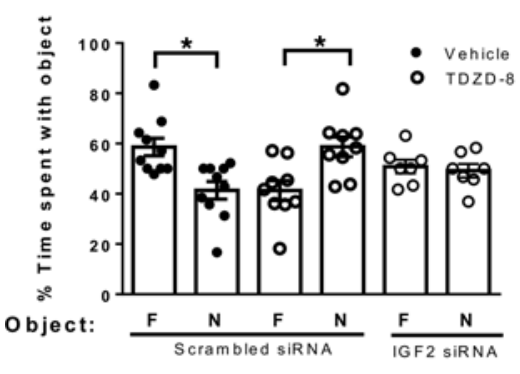

D

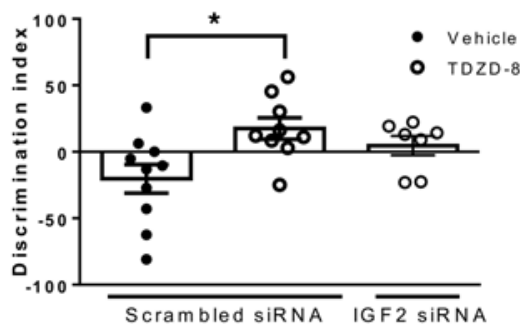

B

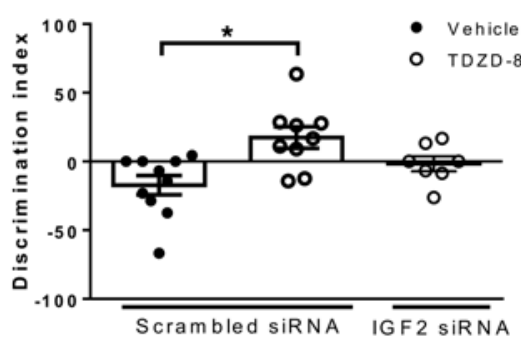

\section{E Coordinate spatial processing}

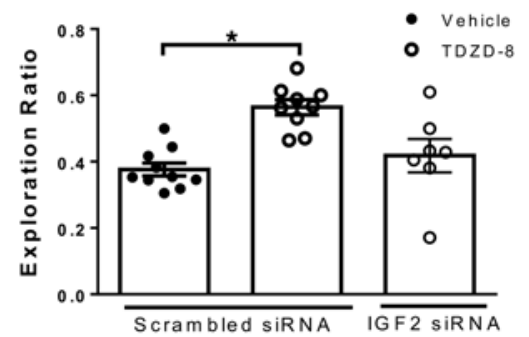

C Temporal order recognition

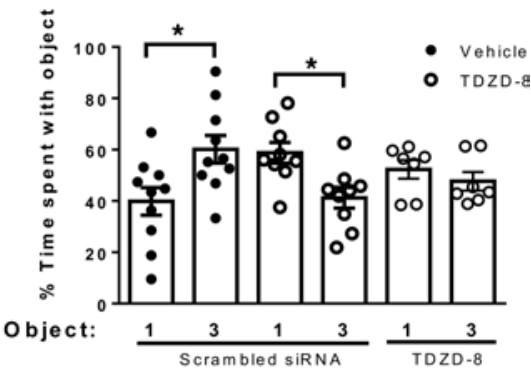

$\mathbf{F}$

Categorical spatial processing

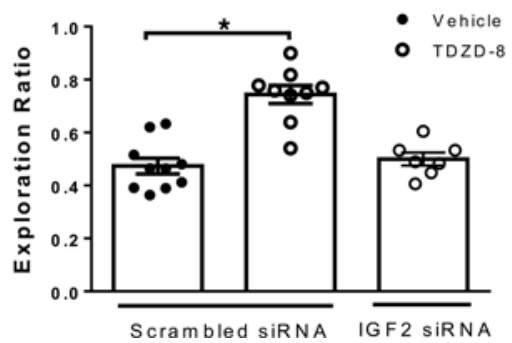

Figure 11. Cognitive performance of TDZD-8-treated Fmr1-/- mice after intranasal administration of insulin-like growth factor-2 (IGF2) siRNA. We tested if intranasal administration of IGF2 siRNA impaired the cognition-repairing effects of the specific GSK3 inhibitor TDZD-8 in Fmr1 ${ }^{-1-}$ mice in novel object recognition, temporal ordering, and coordinate and categorical spatial processing. Mice were treated intranasally with scrambled siRNA or IGF2 siRNA ( $10 \mu \mathrm{g} / \mathrm{mouse} / \mathrm{day})$ administered 3 consecutive days prior to testing and daily throughout the behavioral testing. TDZD-8 (5 mg/kg; i.p.) or vehicle was administered 1 hour prior to behavioral testing. (A) $F \mathrm{Fr}^{-1-}$ mice treated with scrambled siRNA displayed a deficit in novel object recognition that was rescued by treatment with TDZD-8 (vehicle: $n=10 ; \mathrm{t}_{(18)}=3.45,{ }^{*} P<0.01$; TDZD-8: $n=9 ; \mathrm{t}_{(16)}=3.12$, ${ }^{*} P<0.01$ ). Treatment with IGF2 siRNA diminished the improvement in novel object recognition induced by treatment with TDZD-8 $\left(n=7 ; t_{(12)}=0.43\right.$, n.s.). (B) Discrimination index is shown for novel object recognition (one-way ANOVA: $\left.F_{(2,25)}=6.41, P<0.01\right)\left({ }^{*} P<0.01\right.$ compared with scrambled siRNA- and vehicle-treated $\mathrm{Fmr1}^{-1-}$ mice). (C) $\mathrm{Fmr1}^{-1-}$ mice treated with scrambled siRNA displayed a deficit in temporal ordering that was rescued by treatment with TDZD-8 (scrambled siRNA: $n=10 ; \mathrm{t}_{(18)}=2.67,{ }^{*} P<0.05$; TDZD-8: $n=9 ; \mathrm{t}_{(16)}=3.09,{ }^{*} P<0.01$ ). 1, first object presented; 3, most recent object presented. IGF2 siRNA diminished the improvement in temporal ordering induced by treatment with TDZD-8 $\left(n=7 ; t_{(12)}=0.90, n .5\right.$.). (D) Discrimination index is shown for temporal ordering (one-way ANOVA: $\left.F_{(2,25)}=4.68, P<0.05\right)\left({ }^{*} P<0.05\right.$ compared with scrambled siRNA- and vehicle-treated $F m r 1^{-/-}$mice). (E) Fmr1/- mice displayed impaired coordinate spatial processing $(n=10)$ that was rescued by treatment with TDZD-8 ( $n=9)$, and the TDZD-8-induced improvements were blocked by IGF2 siRNA $(n=7)$ treatment $\left(F_{(2.25)}=11.56, P<0.01\right)\left({ }^{*} P<0.01\right.$ compared with scrambled siRNA- and vehicle-treated $\mathrm{Fmr1}^{-1-}$ mice). (F) $\mathrm{Fmr1}^{-1-}$ mice displayed impaired categorical spatial processing $(n=10)$ that was restored by treatment with TDZD-8 $(n=9)$, and the TDZD-8-induced improvements were blocked by IGF2 siRNA $(n=7)$ treatment (one-way ANOVA: $\left.F_{(2,25)}=24.39, P<0.01\right)\left({ }^{*} P<0.01\right.$ compared with scrambled siRNA- and vehicle-treated $\mathrm{Fmr1}^{-1-}$ mice). Values are means $\pm \mathrm{SEM}$. Each symbol represents the value from an individual mouse.

synthesized in the Martinez laboratory (52) and administered (5 mg/kg; i.p.) in 5\% Tween $80,5 \%$ DMSO in saline. ON-TARGETplus Mouse HDAC1 siRNA SMARTpool (10 $\mu \mathrm{g} / \mathrm{mouse} /$ day; L-040287-02; GE Healthcare Dharmacon Inc.), ON-TARGETplus Mouse HDAC2 siRNA SMARTpool (10 $\mu \mathrm{g} / \mathrm{mouse} /$ day; L-046158-00; GE Healthcare Dharmacon Inc.), ON-TARGETplus Mouse HDAC3 siRNA SMARTpool (10 $\mu \mathrm{g} / \mathrm{mouse} /$ day; L-043553-02; GE Healthcare Dharmacon Inc.), ON-TARGETplus Mouse HDAC4 siRNA SMARTpool (10 $\mu \mathrm{g}$ /mouse/day; L-043626-00; GE Healthcare Dharmacon Inc.), and ON-TARGETplus Mouse IGF2 (16002) siRNA (10 $\mu \mathrm{g} / \mathrm{mouse} /$ day; J-043709-09; GE Healthcare Dharmacon Inc.) were administered intranasally for 3 days prior to behavioral testing and daily throughout the behavioral testing. DDX vehicle or ON-TARGETplus Nontargeting siRNA (D-001810-02; GE Helathcare Dharmacon Inc.) was given to matched mice. Mouse BDNF (gene ID 12064) siRNA SMARTpool (1, 3, or $10 \mu \mathrm{g} /$ mouse; M-042566-01; GE Healthcare Dharmacon Inc.) was administered intranasally 48 hours prior to behavioral testing. IGF2 ( $1 \mu \mathrm{g} / \mathrm{mouse} /$ day; 792-MG; R\&D systems; in PBS) was administered intranasally in each nostril ( $5 \mu 1 /$ nostril), 24 hours and 1 hour prior to behavioral testing. Scrambled 
siRNA (Ambion) was used as control in siRNA experiments. Scrambled siRNA coupled to fluorescein was used to detect localization by measuring fluorescence in homogenates of hippocampus and cerebellum after intranasal administration.

\section{Behavioral assays}

For all behavioral assessments, mice were acclimated to the testing room for 30 minutes before testing, the sessions were filmed, a white noise generator (model SS-2000G/F-AMZ, Homedics Inc.) (55 dB) was used, and each object was cleaned with $70 \%$ ethanol between each test session. Object exploration was quantified as time spent exploring the objects, defined as the mouse sniffing or touching an object with its nose, vibrissa, mouth, or forepaws. Time spent exploring each object was obtained from videos blind to the treatment.

Novel object recognition. For this and temporal ordering, objects in duplicate $(4-6 \mathrm{~cm}$ diameter $\times 2-6 \mathrm{~cm}$ height) were used in a Plexiglas box $(26 \mathrm{~cm}$ long $\times 20 \mathrm{~cm}$ wide $\times 16 \mathrm{~cm}$ tall). Each mouse individually was allowed to explore 2 identical copies of object 1 in the arena for 5 minutes. Subsequently, the mouse was placed for 5 minutes in an opaque holding container, and then the mouse was placed in the arena for 5 minutes containing one new copy of the familiar $(\mathrm{F})$ object and a novel $(\mathrm{N})$ object. The percent of object exploration time that the mouse spent with each object was measured. More time spent exploring the novel object than the familiar object indicates normal memory processing. The discrimination index was calculated as percent times: (exploration of novel object - exploration of familiar object)/total time exploring objects $\times 100$.

Temporal ordering. This task used 3 new sets of objects (objects 1,2,3) different from the novel object task, as previously described $(30,35)$. Each mouse individually was allowed to explore 2 identical copies of object 1, 2, and 3 spaced by 5 -minute periods in an opaque holding container. For the test session, each mouse individually was allowed to explore an unused object 1 and an unused object 3 for 5 minutes. The percent of object exploration time that the mouse spent with each object was measured. Normal temporal order memory is exhibited by mice spending more time exploring the first object presented (object 1) than the most recent object presented (object 3). The discrimination index was calculated as time of: (exploration of object 1 exploration of object 3)/total time exploring objects $\times 100$.

Coordinate and categorical spatial processing. The coordinate spatial processing task involved, first, a habituation session, during which each mouse individually was allowed to explore for 15 minutes 2 different objects separated by $45 \mathrm{~cm}$ in the arena. After 5 minutes in an opaque container, each mouse individually was allowed to explore for 5 minutes the same 2 objects that were separated this time by only $30 \mathrm{~cm}$. Mice that remember the distance between objects display increased exploration of the objects during the test session compared with the last 5 minutes of the habituation phase. The categorical spatial processing task was identical to the coordinate spatial processing task, except that during the test phase, the positions of the objects had been switched, while remaining separated by $45 \mathrm{~cm}$. Mice that remembered the locations of the objects displayed increased exploration of the objects during the test session compared with the last 5 min utes of the habituation phase. The exploration ratio was calculated as: (exploration time during the 5-minute test session)/(exploration time during the 5 -minute test session + exploration time during the last 5 minutes of the habituation session). Increased exploration during the 5-minute test session compared with the last 5 minutes of the habituation session results in an exploration ratio $>0.5$.

$\mathrm{IHC}$

After behavioral testing, mice were anesthetized with ketamine $(100 \mathrm{mg} / \mathrm{kg})$ and xylazine $(10 \mathrm{mg} / \mathrm{kg})$ and transcardially perfused with $0.9 \% \mathrm{NaCl}$ and $4 \%$ paraformaldehyde (PFA; catalog P614, Sigma-Aldrich). Brains were removed and placed in $4 \%$ PFA overnight at $4^{\circ} \mathrm{C}$ and stored in $30 \%$ sucrose and $0.02 \%$ sodium azide in phosphate buffer ( $\mathrm{pH}$ 7.4) until sectioning. Slices of $40 \mu \mathrm{m}$ were prepared using a cryostat (Leica CM1850). Slices were washed in phosphate-buffered saline (PBS, $0.01 \mathrm{M}, \mathrm{pH} 7.5$ ) 3 times for 10 minutes each, rinsed in PBS containing $0.1 \%$ Triton-X for 10 minutes, and blocked in PBS containing 5\% normal goat serum for 30 minutes. Sections were rinsed in PBS for 5 minutes and incubated overnight in primary antibody of anti-GSK3ß 1:250 (catalog 610202, BD Biosciences); anti-HDAC1 1:50 (catalog ab19845, Abcam); antiHDAC2 1:100 (catalog ab16032, Abcam); anti-HDAC3 1:100 (catalog ab16047, Abcam); and anti-HDAC4 1:50 (catalog 2072, Cell Signaling). Sections were washed in PBS 3 times for 10 minutes each, incubated with goat anti-rabbit biotinylated secondary antibody (1:100; catalog 4050-05, Southern Biotech) for 1 hour, washed 3 times for 10 minutes each in PBS, and incubated in a streptavidin-biotin complex solution (ABC Kit, Vector Laboratories) for 1 hour. Sections were washed 3 times for 10 minutes each with PBS and were 
incubated in diaminobenzidine (DAB; catalog SK4100, Vector Laboratories) in phosphate buffer (PB, 0.1 $\mathrm{M}, \mathrm{pH}$ 7.4) for 3 minutes. Slices were washed in PB for 10 minutes, mounted, dehydrated, and cover-slipped using Eukitt (catalog NC9068612, Thermo Fisher Scientific) as mounting medium. Images were acquired using a light microscope Evos XL Core (Invitrogen). Quantification of the number of positive cells for each antigen was performed by blind counting of the total number of cells stained positive for the antigen in at least 5 slices from each mouse. For each mouse, average values from all replicates were determined. The final results are expressed as number of labeled neurons for each antigen.

\section{ELISA}

Hippocampi were rapidly dissected in ice-cold $\mathrm{PBS}$, snap-frozen, and stored at $-80^{\circ} \mathrm{C}$; they were later homogenized in ice-cold lysis buffer containing $20 \mathrm{mM}$ Tris- $\mathrm{HCl}$, pH 7.4, $150 \mathrm{mM} \mathrm{NaCl}, 1 \mathrm{mM}$ EDTA, $1 \mathrm{mM}$ EGTA, $1 \%$ Triton-100, $10 \mu \mathrm{g} / \mathrm{ml}$ leupeptin, $10 \mu \mathrm{g} / \mathrm{ml}$ aprotinin, $5 \mu \mathrm{g} / \mathrm{ml}$ pepstatin, $1 \mathrm{mM}$ phenylmethanesulfonyl fluoride, $1 \mathrm{mM}$ sodium vanadate, $50 \mathrm{mM}$ sodium fluoride, and $100 \mathrm{nM}$ okadaic acid. The lysates were centrifuged at 17,900 $\mathrm{g}$ for 10 minutes to remove insoluble debris. The protein concentration of hippocampi extract was determined using the Bradford protein assay. IGF2 ELISA was carried out according to the manufacturer's instructions (RayBiotech) using $100 \mu \mathrm{g}$ protein.

\section{RNAseq analysis}

RNAseq analysis was performed utilizing CANEapp, an application for comprehensive analysis of RNA seq data. CANEapp is a freely available Java application generated at the University of Miami that allows a completely automated analysis of RNAseq data for the detection of differentially expressed genes (http://psychiatry.med.miami.edu/research/laboratory-of-translational-rna-genomics/CANE-app) (53). In more detail, paired-end reads generated from sequencing on the Illumina Hiseq2000 were trimmed off the adaptor sequences using a custom script and were aligned to the human genome reference GRCh37 using TopHat 2.0.9. Transcriptome for each sample was assembled using Cufflinks, which also provided transcripts abundance in fragments per kilobase of exon per million fragments mapped (FPKM), and transcripts assemblies were merged using Cuffmerge. Differential gene expression analysis was performed using Cuffdiff. Genes were annotated according to the ENSEMBL classification, and genes that were not annotated as proteins coding genes, lincRNAs, or antisense RNAs were filtered out. The data discussed in this publication have been deposited in NCBI's Gene Expression Omnibus (GEO) and are accessible through GEO Series accession number GSE83217.

\section{qPCR}

Total RNA from brain regions was isolated by TRIzol extraction (Invitrogen). RNA (500 ng) was converted to cDNA using ImProvII reverse transcriptase (Promega) according to the manufacturer's instructions. Quantitative changes in the mRNA levels were determined by the qTower 2.2 from AnalytikJena real-time PCR system (Biometra) using SYBR green according to the manufacturer's instructions using $12.5 \mathrm{ng}$ of cDNA. Quantification was made with 2- $\Delta \Delta \mathrm{Ct}$ method using GAPDH as the housekeeping gene. Primers are shown in Supplemental Table 2.

\section{Statistics}

The data were analyzed with independent 2-tailed sample $t$ tests, one-way or 2-way ANOVA followed up by Dunnett's post-hoc tests (as reported in figure legends). All bars and error bars represent the mean \pm SEM, and significance was set at $P<0.05$.

\section{Study approval}

All procedures with mice were reviewed and approved by the University of Miami Institutional Animal Care and Use Committee, Miami, Florida, and were carried out in accordance with NIH regulations.

\section{Author contributions}

MP and YC performed the experiments; DV, MM, and MAF performed the data analysis of the RNA sequencing; HEF and AM provided GSK3 inhibitors; and RSJ and EB designed the experiments and wrote the manuscript. 


\section{Acknowledgments}

The authors thank Emma Perez-Costas and Miguel Melendez-Ferro for their valuable contributions to the IHC procedures. We thank the John P. Hussman Institute for Human Genomics personnel within the Center for Genome Technology for their help and support of this project. This research was supported by grants from the NIMH (MH038752, MH090236, MH095380, MH104656) and a NARSAD Distinguished Investigator grant from the Brain \& Behavior Research Foundation.

Address correspondence to: Richard S. Jope, Miller School of Medicine, University of Miami, 1011 NW 15th Street, Gautier Building room 416, Miami, Florida 33136 USA. Phone: 305.243.0262; E-mail: rjope@med.miami.edu.

1. Woodgett JR. Judging a protein by more than its name: GSK-3. Sci STKE. 2001;2001(100):re12.

2. Beurel E, Grieco SF, Jope RS. Glycogen synthase kinase-3 (GSK3): regulation, actions, and diseases. Pharmacol Ther. 2015;148:114-131.

3. McManus EJ, et al. Role that phosphorylation of GSK3 plays in insulin and Wnt signalling defined by knockin analysis. EMBO J. 2005;24(8):1571-1583.

4. Peineau S, et al. LTP inhibits LTD in the hippocampus via regulation of GSK3beta. Neuron. 2007;53(5):703-717.

5. Bradley CA, et al. A pivotal role of GSK-3 in synaptic plasticity. Front Mol Neurosci. 2012;5:13.

6. King MK, Pardo M, Cheng Y, Downey K, Jope RS, Beurel E. Glycogen synthase kinase-3 inhibitors: Rescuers of cognitive impairments. Pharmacol Ther. 2014;141(1):1-12.

7. Mines MA, Jope RS. Glycogen synthase kinase-3: a promising therapeutic target for fragile x syndrome. Front Mol Neurosci. 2011;4:35.

8. Eldar-Finkelman H, Martinez A. GSK-3 Inhibitors: Preclinical and Clinical Focus on CNS. Front Mol Neurosci. 2011;4:32.

9. Alarcón JM, et al. Chromatin acetylation, memory, and LTP are impaired in CBP+/- mice: a model for the cognitive deficit in Rubinstein-Taybi syndrome and its amelioration. Neuron. 2004;42(6):947-959.

10. Levenson JM, O'Riordan KJ, Brown KD, Trinh MA, Molfese DL, Sweatt JD. Regulation of histone acetylation during memory formation in the hippocampus. J Biol Chem. 2004;279(39):40545-40559.

11. Yeh SH, Lin CH, Gean PW. Acetylation of nuclear factor-kappaB in rat amygdala improves long-term but not short-term retention of fear memory. Mol Pharmacol. 2004;65(5):1286-1292.

12. Fischer A, Sananbenesi F, Wang X, Dobbin M, Tsai LH. Recovery of learning and memory is associated with chromatin remodelling. Nature. 2007;447(7141):178-182.

13. Fischer A, Sananbenesi F, Mungenast A, Tsai LH. Targeting the correct HDAC(s) to treat cognitive disorders. Trends Pharmacol Sci. 2010;31(12):605-617.

14. Penney J, Tsai LH. Histone deacetylases in memory and cognition. Sci Signal. 2014;7(355):re12.

15. Guan JS, et al. HDAC2 negatively regulates memory formation and synaptic plasticity. Nature. 2009;459(7243):55-60.

16. Gräff J, et al. An epigenetic blockade of cognitive functions in the neurodegenerating brain. Nature. 2012;483(7388):222-226.

17. Hanson JE, et al. Histone deacetylase 2 cell autonomously suppresses excitatory and enhances inhibitory synaptic function in CA1 pyramidal neurons. J Neurosci. 2013;33(14):5924-5929.

18. Morris MJ, Mahgoub M, Na ES, Pranav H, Monteggia LM. Loss of histone deacetylase 2 improves working memory and accelerates extinction learning. J Neurosci. 2013;33(15):6401-6411.

19. McQuown SC, et al. HDAC3 is a critical negative regulator of long-term memory formation. J Neurosci. 2011;31(2):764-774.

20. Kim MS, et al. An essential role for histone deacetylase 4 in synaptic plasticity and memory formation. J Neurosci. 2012;32(32):10879-10886.

21. Sando R, Gounko N, Pieraut S, Liao L, Yates J, Maximov A. HDAC4 governs a transcriptional program essential for synaptic plasticity and memory. Cell. 2012;151(4):821-834.

22. Falkenberg KJ, Johnstone RW. Histone deacetylases and their inhibitors in cancer, neurological diseases and immune disorders. Nat Rev Drug Discov. 2014;13(9):673-691.

23. Abend A, Kehat I. Histone deacetylases as therapeutic targets--from cancer to cardiac disease. Pharmacol Ther. 2015;147:55-62

24. Gherardini L, Bardi G, Gennaro M, Pizzorusso T. Novel siRNA delivery strategy: a new "strand" in CNS translational medicine? Cell Mol Life Sci. 2014;71(1):1-20.

25. Gomes MJ, Martins S, Sarmento B. siRNA as a tool to improve the treatment of brain diseases: Mechanism, targets and delivery. Ageing Res Rev. 2015;21:43-54.

26. Mistry A, Stolnik S, Illum L. Nanoparticles for direct nose-to-brain delivery of drugs. Int J Pharm. 2009;379(1):146-157.

27. Biddlestone-Thorpe L, et al. Nanomaterial-mediated CNS delivery of diagnostic and therapeutic agents. Adv Drug Deliv Rev. 2012;64(7):605-613.

28. Santos AR, Kanellopoulos AK, Bagni C. Learning and behavioral deficits associated with the absence of the fragile X mental retardation protein: what a fly and mouse model can teach us. Learn Mem. 2014;21(10):543-555.

29. Yuskaitis CJ, Mines MA, King MK, Sweatt JD, Miller CA, Jope RS. Lithium ameliorates altered glycogen synthase kinase-3 and behavior in a mouse model of fragile X syndrome. Biochem Pharmacol. 2010;79(4):632-646.

30. King MK, Jope RS. Lithium treatment alleviates impaired cognition in a mouse model of fragile X syndrome. Genes Brain Behav. 2013;12(7):723-731.

31. Franklin AV, King MK, Palomo V, Martinez A, McMahon LL, Jope RS. Glycogen synthase kinase-3 inhibitors reverse deficits in long-term potentiation and cognition in fragile X mice. Biol Psychiatry. 2014;75(3):198-206. 
32. Kaidanovich-Beilin O, Milman A, Weizman A, Pick CG, Eldar-Finkelman H. Rapid antidepressive-like activity of specific glycogen synthase kinase-3 inhibitor and its effect on beta-catenin in mouse hippocampus. Biol Psychiatry. 2004;55(8):781-784.

33. Alonso M, et al. BDNF-triggered events in the rat hippocampus are required for both short- and long-term memory formation. Hippocampus. 2002;12(4):551-560.

34. Bekinschtein P, Cammarota M, Medina JH. BDNF and memory processing. Neuropharmacology. 2014;76 Pt C:677-683.

35. Pardo M, et al. Impairments in cognition and neural precursor cell proliferation in mice expressing constitutively active glycogen synthase kinase-3. Front Behav Neurosci. 2015;9:55.

36. Chen DY, et al. A critical role for IGF-II in memory consolidation and enhancement. Nature. 2011;469(7331):491-497.

37. Ouchi Y, et al. Reduced adult hippocampal neurogenesis and working memory deficits in the Dgcr8-deficient mouse model of 22q11.2 deletion-associated schizophrenia can be rescued by IGF2. J Neurosci. 2013;33(22):9408-9419.

38. Lee Y, Lee YW, Gao Q, Lee Y, Lee HE, Ryu JH. Exogenous insulin-like growth factor 2 administration enhances memory consolidation and persistence in a time-dependent manner. Brain Res. 2015;1622:466-473.

39. Shahmoradi A, Radyushkin K, Rossner MJ. Enhanced memory consolidation in mice lacking the circadian modulators Sharp1 and -2 caused by elevated Igf2 signaling in the cortex. Proc Natl Acad Sci USA. 2015;112(27):E3582-E3589.

40. Senn C, Hangartner C, Moes S, Guerini D, Hofbauer KG. Central administration of small interfering RNAs in rats: a comparison with antisense oligonucleotides. Eur J Pharmacol. 2005;522(1-3):30-37.

41. Sheng G, et al. Hypothalamic huntingtin-associated protein 1 as a mediator of feeding behavior. Nat Med. 2006;12(5):526-533

42. Salahpour A, Medvedev IO, Beaulieu JM, Gainetdinov RR, Caron MG. Local knockdown of genes in the brain using small interfering RNA: a phenotypic comparison with knockout animals. Biol Psychiatry. 2007;61(1):65-69.

43. Bortolozzi A, et al. Selective siRNA-mediated suppression of 5-HT1A autoreceptors evokes strong anti-depressant-like effects Mol Psychiatry. 2012;17(6):612-623.

44. Kanazawa T, Morisaki K, Suzuki S, Takashima Y. Prolongation of life in rats with malignant glioma by intranasal siRNA/drug codelivery to the brain with cell-penetrating peptide-modified micelles. Mol Pharm. 2014;11(5):1471-1478.

45. Lu Y, Christian K, Lu B. BDNF: a key regulator for protein synthesis-dependent LTP and long-term memory? Neurobiol Learn Mem. 2008;89(3):312-323

46. Gottmann K, Mittmann T, Lessmann V. BDNF signaling in the formation, maturation and plasticity of glutamatergic and GABAergic synapses. Exp Brain Res. 2009;199(3-4):203-234.

47. Alberini CM, Chen DY. Memory enhancement: consolidation, reconsolidation and insulin-like growth factor 2. Trends Neurosci. 2012;35(5):274-283

48. Wise TL. Changes in insulin-like growth factor signaling alter phenotypes in Fragile X Mice. Genes Brain Behav. 2017;16(2):241-249.

49. Eom TY, Jope RS. Blocked inhibitory serine-phosphorylation of glycogen synthase kinase-3alpha/beta impairs in vivo neural precursor cell proliferation. Biol Psychiatry. 2009;66(5):494-502.

50. Polter A, et al. Deficiency in the inhibitory serine-phosphorylation of glycogen synthase kinase-3 increases sensitivity to mood disturbances. Neuropsychopharmacology. 2010;35(8):1761-1774.

51. Plotkin B, Kaidanovich O, Talior I, Eldar-Finkelman H. Insulin mimetic action of synthetic phosphorylated peptide inhibitors of glycogen synthase kinase-3. J Pharmacol Exp Ther. 2003;305(3):974-980.

52. Martinez A, Alonso M, Castro A, Pérez C, Moreno FJ. First non-ATP competitive glycogen synthase kinase 3 beta (GSK-3beta) inhibitors: thiadiazolidinones (TDZD) as potential drugs for the treatment of Alzheimer's disease. JMed Chem. 2002;45(6):1292-1299.

53. Velmeshev D, Lally P, Magistri M, Faghihi MA. CANEapp: a user-friendly application for automated next generation transcriptomic data analysis. BMC Genomics. 2016;17:49. 